International Journal of Modern Physics B Vol. 27, No. 32 (2013) 1399001 (20 pages) (C) World Scientific Publishing Company DOI: $10.1142 / S 0217979213990014$

\title{
AUTHOR INDEX Volume 27
}

Abadyan, M., see Beni, Y. T.

Abd-Elnabi, S. \& Osman, K. I., Controllability of electromagnetically induced transparency in a doppler broadened fourlevel atomic system

Abdel-Aty, M., see Metwally, N. Abdel-Rahman, E., see Aly, A. H. Abou Zied, M., see Ebnalwaled, A. A.

Acharya, S. \& Mukherjee, K., Heat transport in a three dimensional slab geometry and the temperature profile of Ingen-Hausz's experiment

Acharya, S. \& Mukherjee, K., Heat transport in a threedimensional slab geometry and the temperature profile of Ingen-Hausz's experiment

Adebambo, P. O., see Adetunji, B. I

Adebayo, G. A., see Adetunji, B.

Adesso, G., see Girolami, D.

Adetunji, B. I., Adebambo, P. O., Akinlami, J. O. \& Adebayo, G. A., Electronic and elastic properties of zinc-blende $\mathrm{MgSe}$

Adhikari, N. P., see Thapa, S. K.

Agrawal (Garg), N., Ghosh, S. \& Sharma, M., Electron optics with dirac fermions: electron transport in monolayer and bilayer graphene through magnetic barrier and their superlattices

Ahmad, I., see Shah, A.

Ahmad, K., see Noor, K. I.

Ahmadi, M. T., see Kang, E. S.

Ahmadian, M. T., see Moeenfard,

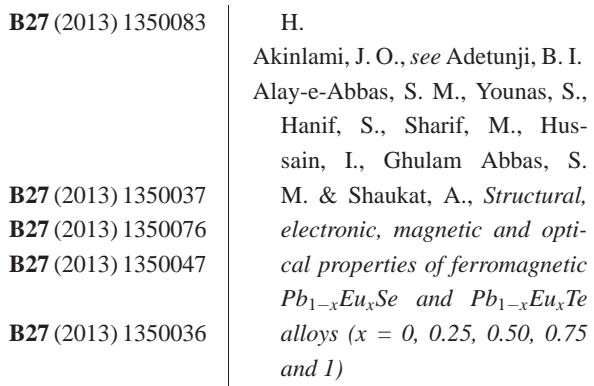

M. \& Shaukat, A., Structural, electronic, magnetic and optical properties of ferromagnetic $P b_{1-x} E u_{x} S e$ and $P b_{1-x} E u_{x} T e$ alloys $(x=0,0.25,0.50,0.75$ and 1)

B27 (2013) 1350027

B27 (2013) 1350027 B27 (2013) 1345020

B27 (2013) 1350027 B27 (2013) 1350023

B27 (2013) 1341003 B27 (2013) 1350020 B27 (2013) 1250203 B27 (2013) 1350077
Albarakati, A., see Wang, D.

Albayrak, E., The quantum refrigerator in a two-qubit $\mathrm{XXZ}$ Heisenberg model

Albertini, G., Calbucci, V., Cardone, F., Fattorini, G., Mignani, R., Petrucci, A., Ridolfi, F. \& Rotili, A., Evidence of alpha emission from compressed steel bars

Ali, R., Khanata, R., Amin, B., Murtaza, G. \& Omran, S. B., Structural, elastic, electronic, chemical bonding and optical properties of $\mathrm{M}_{2} \mathrm{Se}$ ( $\mathrm{M}=\mathrm{Li}, \mathrm{Na}$, $K, R b)$ through first principle study

Aliev, M. A. \& Yu. Kuzminykh, N., Microphase separation in polydisperse graft copolymers

Almanza, O. A. M., see Rendón Ramírez, J. M.

Aly, A. H., Mehaney, A. \& AbdelRahman, E., Study of physical parameters on the properties of phononic band gaps

Amico, L., see Sarandy, M. S.

Amin, B., see Ali, R.

Amin, B., see Semari, F.

An, Z.-W., see Wang, X.-X.
B27 (2013) 1350008 B27 (2013) 1350027

B27 (2013) 1350100 B27 (2013) 1330016

B27 (2013) 1350055

B27 (2013) 1350124

B27 (2013) 1350170

B27 (2013) 1350189

B27 (2013) 135017

B27 (2013) 1350047 B27 (2013) 1345030 B27 (2013) 1350170 B27 (2013) 1350082 B27 (2013) 1350050 
Anderson, D., see Cattani, F.

Andreev, P. A., First principles derivation of NLS equation for BEC with cubic and quintic nonlinearities at nonzero temperature: dispersion of linear waves

Angelakis, D. \& Mancini, S., Fluctuations assisted stationary entanglement in driven quantum systems

Angilella, G. G. N., see March, N. $\mathrm{H}$.

Anwar, S., see Kang, E. S.

Apollaro, T. J. G., Lorenzo, S. \& Plastina, F., Transport of quantum correlations across a spin chain

Arham, H. Z., see Lee, W.-C.

Armstrong, R. W., Elban, W. L. \& Walley, S. M., Elastic, plastic, cracking aspects of the hardness of materials

Asadpour, S. H., Soltani, A., Majd, A. E. \& Soleimani, H. R., Far infrared photo detector based on electromagnetically induced transparency

Aslantaş, A., see Yurtseven, H.

Ateser, E., Ozisik, H. B., Deligoz, E. \& Colakoglu, K., The firstprinciples stability study of $P d C$ and $\mathrm{CdC}$ compounds

Audzijonis, A. \& Sereika, R., Electronic structure and electron charge density in the interatomic bonds of BiSBr and BiSeBr crystals

Azadehranjbar, S., Karimzadeh, F., Enayati, M. H. \& Mahmoodi, N., Properties of bulk $\mathrm{Fe}-\mathrm{Ni} / \mathrm{CNT}$ nanocomposites prepared by mechanical milling and sintering

Baaouague, K., see Ouchene, S.

Bagayoko, D., see Malozovsky, Y.

Bagayoko, D., see Tan, L.

Bahari, A., Gholipur, R. \& Derakhshi, M., The investigation on stability and structural properties of cohesion-based nanostructures material

Bai, M.-Q., see Peng, J.-Y.

Bakhshayeshi, A., see Taghavi Mendi, R.
B27 (2013) 1330003

B27 (2013) 1350017

B27 (2013) 1345037

B27 (2013) 1330021

B27 (2013) 1350077

B27 (2013) 1345035

B27 (2013) 1330014

B27 (2013) 1330004

B27 (2013) 1350004

B27 (2013) 1350125

B27 (2013) 1350016

B27 (2013) 1350122

B27 (2013) 1350102

B27 (2013) 1350142

B27 (2013) 1362013

B27 (2013) 1362017

B27 (2013) 1350153

B27 (2013) 1350137

B27 (2013) 1350096
Barbosa, I. M., see Caetano, R.

Bassan, M., Buonomo, B., Cavallari, G., Coccia, E., D’Antonio, S., Fafone, V., Foggetta, L. G., Ligi, C., Marini, A., Mazzitelli, G., Modestino, G., Pizzella, G., Quintieri, L., Ronga, F. \& Valente, P., Measurement of the thermal expansion coefficient of an Al-Mg alloy at ultra-low temperatures

Behera, D., see Sahoo, M.

Belevtsev, B. I., Yu. Beliayev, E. \& Kolesnichenko, Y. A., $1 D$ and $2 D$ quantum interference effects in electron transport in Au film Belkhir, H., see Ouchene, S.

Bellaiche, L., see Wang, D.

Bellomo, B., see Lo Franco, R.

Beni, Y. T. \& Abadyan, M., Use of strain gradient theory for modeling the size-dependent pull-in of rotational nano-mirror in the presence of molecular force

Bennaceur, R., see Rabhi, A.

Beran, Z. \& Čelikovský, S., Generalized semiflows and chaos in multivalued dynamical systems

Bergholtz, E. J. \& Liu, Z., Topological flat band models and fractional Chern insulators

Beryani Nezafat, N., see Taghavi Mendi, R.

Bhalla, V., Kumar, R., Tripathy, C. \& Singh, D., Mechanical and thermal properties of praseodymium monopnictides: An ultrasonic study

Bludov, Y. V., Ferreira, A., Peres, N. M. R. \& Vasilevskiy, M. I., A primer on surface plasmonpolaritons in graphene

Bogolubov Jr, N. N. \& Prykarpatsky, Y. A., The Marsden-Weinstein reduction structure of integrable dynamical systems and a generalized exactly solvable quantum superradiance model

Bohr, H. G. \& Malik, F. B., Coherence, energy and charge transfers in de-excitation pathways of electronic excited state of biomolecules in photosynthesis

Boochani, A., see Taghavi Mendi,
B27 (2013) 1350089

B27 (2013) 1350119

B27 (2013) 1350099

B27 (2013) 1350026

B27 (2013) 1350142

B27 (2013) 1330016

B27 (2013) 1345053

B27 (2013) 1350083

B27 (2013) 1350058

B27 (2013) 1392001

B27 (2013) 1330017

B27 (2013) 1350096

B27 (2013) 1350116

B27 (2013) 1341001

B27 (2013) 1350002

B27 (2013) 1347007 
$\mathrm{R}$

Bose, I. \& Pal, A. K., Quantum discord, decoherence and quantum phase transition

Bouhemadou, A., see Semari, F. Brajczewska, M., see Rabhi, A. Buonomo, B., see Bassan, M. Busch, T., see Giorgi, G. L.

Caetano, R., Hoyer, Y. D., Barbosa, I. M., Grigorov, K. G. \& Sismanoglu, B. N., Radial measurements of gas discharge parameters of atmospheric pressure microplasma

Cai, C. Z., see Xiao, T. T.

Calbucci, V., see Albertini, G.

Canosa, N., Ciliberti, L. \& Rossignoli, R., Quantum discord and related measures of quantum correlations in finite $X Y$ chains

Cao, H., see Chung, T. F.

Cao, H.-L., see Li, T.-H.

Cao, J. C., see Wang, C.

Cao, J. X., see Yang, Q.

Cao, M., see Liu, H.

Cardona, R., see Rendón Ramírez, J. M.

Cardone, F., see Albertini, G.

Castellanos, E. \& Matos, T., Klein-Gordon fields and BoseEinstein condensates: Thermal bath contributions

Cattani, F., Kim, A., Lisak, M. \& Anderson, D., Interactions of electromagnetic radiation with Bose-Einstein condensates: manipulating ultra-cold atoms with light

Cavallari, G., see Bassan, M.

Çetinbaş İşeri, E., see Yurtseven, $\mathrm{H}$.

Čelikovský, S., see Beran, Z.

Cha, M.-C., Asymmetric twocomponent hard-core Boson mixtures in a one-dimensional optical lattice

Cha, M.-C., Phase transition of Bosons driven by a staggered gauge field in an optical lattice

Chacón-Acosta, G., see Santiago, J. A.

Chakrabarti, B., see Haldar, S. K.

Chatterjee, A., see Mukhopadhyay, $\mathrm{S}$.

Chen, B., see Chen, T.-P.
B27 (2013) 1350096

B27 (2013) 1345042

B27 (2013) 1350082

B27 (2013) 1350058

B27 (2013) 1350119

B27 (2013) 1345034

B27 (2013) 1350089

B27 (2013) 1362040

B27 (2013) 1350124

B27 (2013) 1345033

B27 (2013) 1341002

B27 (2013) 1350148

B27 (2013) 1350105

B27 (2013) 1350138

B27 (2013) 1350174

B27 (2013) 1350171

B27 (2013) 1350124

B27 (2013) 1350060

B27 (2013) 1330003

B27 (2013) 1350119

B27 (2013) 1350063

B27 (2013) 1392001

B27 (2013) 1362002

B27 (2013) 1362037

B27 (2013) 1350043

B27 (2013) 1350048

B27 (2013) 1350098

B27 (2013) 1362009
Chen, C. P., see Min, Y.

Chen, C., see Tong, M.-W.

Chen, C.-J., see Chen, T.-P.

Chen, F., see Wang, X.-Y.

Chen, H. Z., Kao, M. C. \& Young, S. L., Magnetic and structural transition properties of niobium-doped $\mathrm{BiFeO}_{3}$ thin films

Chen, H., Xu, W., Wang, W., Wang, R. \& Shi, C., A nonlinear viscoelastic-plastic rheological model for rocks based on fractional derivative theory

Chen, H.-H., see Cheng, Y.

Chen, H.-Z., see Young, S.-L.

Chen, L., see Yuan, H.-C.

Chen, L.-J., see Yang, M.

Chen, N.-X., see Wang, X.-X.

Chen, N.-X., see Zhang, C.-H.

Chen, T.-P., Wu, K., Wang, S. Z., Li, Q., Chen, B., Tipparaach, U. \& Chen, C.-J., Finite size effect on YBCO/PBCAO and YBCO/PBCGO nanometer multilayers

Chen, X. S., see Duan, H.

Chen, X., see Zhao, H.

Chen, X., Casimir torque on two rotating plates

Chen, X.-X., see Tang, C.-M.

Chen, Y. P., see Chung, T. F.

Chen, Y., see Gui, Z.

Chen, Y., see Liu, H.

Chen, Y., see Wu, Y.

Chen, Y., see Xu, Z.

Chen, Y.-M., Cheng, W., Liao, B. \& Zhang, X., Structural, elastic and electronic properties of oxygen substitution in cubic $\mathrm{Zr}_{3} \mathrm{~N}_{4}$ and $\mathrm{Ti}_{3} \mathrm{~N}_{4}$

Chen, Z., Ho, Y. K., Wang, P. X., Kong, Q., Xie, Y. J., Wang, W. $\& \mathrm{Xu}, \mathrm{J} . \mathrm{J} .$, A formula on phase velocity of waves and application

Cheng, W., see Chen, Y.-M.

Cheng, X., see Wang, H.

Cheng, Y., Chen, H.-H., Xue, F.X., Ji, G.-F. \& Gong, M., Phase transition, elastic and thermodynamic properties of Beryllium via first principles

Cheng, Z., Condensation state of
B27 (2013) 1350081

B27 (2013) 1362008

B27 (2013) 1362009

B27 (2013) 1350196

B27 (2013) 1362003

B27 (2013) 1350149

B27 (2013) 1350130

B27 (2013) 1362006

B27 (2013) 1350120

B27 (2013) 1350006

B27 (2013) 1350050

B27 (2013) 1350147

B27 (2013) 1362009

B27 (2013) 1350178

B27 (2013) 1350086

B27 (2013) 1350066

B27 (2013) 1350040

B27 (2013) 1341002

B27 (2013) 1350007

B27 (2013) 1350174

B27 (2013) 1350021

B27 (2013) 1361003

B27 (2013) 1350095

B27 (2013) 1393001

B27 (2013) 1350095 B27 (2013) 1350061

B27 (2013) 1350130 
ultra-cold Bose atomic gases with noncontact interaction

Chien, C.-C., see Guo, H.

Cho, G.-B., see Kim, N.-H.

Choi, D.-Y., see Kim, N.-H.

Choi, J. R., see Lyu, H.

Choi, J., Choi, M. Y., Chung, M. S. \& Yoon, B.-G., Dynamic transition and resonance in coupled oscillators under symmetry-breaking fields

Choi, M. Y., see Choi, J.

Chou, C.-I. \& Ho, C.-L., Generalized Rayleigh and Jacobi processes and exceptional orthogonal polynomials

Chouahda, Z., see Nemiri, O

Chung, M. S., see Choi, J.

Chung, T. F., Shen, T., Cao, H., Jauregui, L. A., Wu, W., Yu, Q., Newell, D. \& Chen, Y. P., Synthetic graphene grown by chemical vapor deposition on copper foils

Ciliberti, L., see Canosa, N.

Civitarese, O., see Reboiro, M.

Clark, J. W., Khodel, V. A. \& Zverev, M. V., Classical behavior of two-dimensional liquid ${ }^{3} \mathrm{He}$ near a quantum critical point

Clark, J. W., Memorial tribute to Manfred L. Ristig (1935-2011)

Cleri, L. C., see da Silva Jr, M. A.

Coccia, E., see Bassan, M.

Colakoglu, K., see Ateser, E.

Colakoglu, K., see Ozisik, H. B.

Compagno, G., see Lo Franco, R.

Crokidakis, N., see Salmon, O. D. R.

Cui, Y., see Ye, H.

Cui, Y.-M., Wang, Y.-S., Lv, G. \& Guan, R.-H., Qualitative calculation of heat transfer in a cylindrical composite conductor $\mathrm{Cu} / \mathrm{NbTi}$

D'Antonio, S., see Bassan, M.

da Providência, J., see Nishiyama, $\mathrm{S}$.

Da Providência, J., see Rabhi, A.

da Silva Jr, M. A., Serra, R. M. \& Cleri, L. C., Observer invariance of the collapse postulate of quantum mechanics

Dada, O., see Olorunsola, O.
B27 (2013) 1361007

B27 (2013) 1330010

B27 (2013) 1350030

B27 (2013) 1350030

B27 (2013) 1350159

B27 (2013) 1350062 B27 (2013) 1350062

B27 (2013) 1350135 B27 (2013) 1350166 B27 (2013) 1350062

B27 (2013) 1341002 B27 (2013) 1345033 B27 (2013) 1350117

B27 (2013) 1347005

B27 (2013) 1347003

B27 (2013) 1345013

B27 (2013) 1350119

B27 (2013) 1350016

B27 (2013) 1350046

B27 (2013) 1345053

B27 (2013) 1350162 B27 (2013) 1350144

B27 (2013) 1361006 B27 (2013) 1350119

B27 (2013) 1350079

B27 (2013) 1350058

B27 (2013) 1345013 B27 (2013) 1350175
Daniel, M., see Zdravković, S.

Dariescu, C., see Dariescu, M.-A.

Dariescu, M.-A. \& Dariescu, C., Polynomial solutions of Heun equation describing fermions in graphene

Darvishian, A., see Moeenfard, H.

Das, N., Effects of strain coupling and marginal dimensionality in the nature of phase transition in quantum paraelectrics

Datta, A., see Madhok, V.

Davoodi, H. \& Kaatuzian, H., Base composition effects study on NBR current and current gain in SiGe HBT

Davoodi, H. \& Kaatuzian, H., Base composition effects study on NBR current and current gain in SiGe HBT

de Llano, M., see Lomnitz, M.

de Llano, M., see Malik, G. P.

de Llano, M., see Mamedov, T. A.

de Oliveira, T. R., see Sarandy, M. $\mathrm{S}$.

de Sousa, J. R., see Salmon, O. D. R.

Debierre, V., see Durt, T.

Deligoz, E., see Ateser, E.

Deligoz, E., see Ozisik, H. B.

Demircioglu, B., see Mukhopadhyay, $\mathrm{S}$.

Deng, Z., Tsuzuki, K., Miki, M., Felder, B., Hara, S. \& Izumi, M., Flux trapping properties of bulk high-T $T_{c}$ superconductors in static field-cooling magnetization

Derakhshi, M., see Bahari, A.

di Lorenzo, A., Are quantum correlations genuinely quantum?

Di, Z., see Li, M.

Ding, H., see Gong, $\mathrm{P}$

Dong, H.-N., see Liu, J.

Dong, Y. Z., see Duan, H.

Dong, Z. C., see Min, Y.

Dorner, R. \& Vedral, V., Correlations in quantum physics

Dotov, D. G., see Frank, T. D.

Dou, H.-L., see Shao, C.-X.

Du, H., see Wang, C.-Y.

$\mathrm{Du}$, J., see Guo, M.

Du, Q., Hang, C. \& Huang, G., Crossover from spontaneously generated coherence to autler-
B27 (2013) 1350184

B27 (2013) 1350190

B27 (2013) 1350190 B27 (2013) 1350008

B27 (2013) 1350028 B27 (2013) 1345041

B27 (2013) 1350097

B27 (2013) 1392004 B27 (2013) 1347001 B27 (2013) 1347008 B27 (2013) 1347002

B27 (2013) 1345030

B27 (2013) 1350162

B27 (2013) 1345014

B27 (2013) 1350016

B27 (2013) 1350046

B27 (2013) 1350098

B27 (2013) 1362026 B27 (2013) 1350153

B27 (2013) 1345016 B27 (2013) 1350146 B27 (2013) 1350087 B27 (2013) 1362007 B27 (2013) 1350178 B27 (2013) 1350081

B27 (2013) 1345017 B27 (2013) 1350156 B27 (2013) 1350052 B27 (2013) 1350187 B27 (2013) 1350123 
townes splitting in three-level atomic systems

Duan, H., Dong, Y. Z., Lin, Z. P., Zhang, X., Huang, Y. \& Chen, X. S., Energetics of $\mathrm{V}_{\mathrm{Hg}}$-related defects in As-doped $\mathrm{HgCdTe}$

Duan, W.-S., see Yang, Y.

Dufour, J. J., Dufour, X. J. C. \& Vinko, J. D., Pico-chemistry: the possibility of new phases in some hydrogen/metal systems

Dufour, X. J. C., see Dufour, J. J.

Durt, T. \& Debierre, V., Coherent states and the classicalquantum limit considered from the point of view of entanglement

Durt, T., Correlations of decay times of entangled composite unstable systems

Dutta, A., see Nag, T.

Dwivedi, S., see Shripal

Ebnalwaled, A. A. \& Abou Zied, M., Milling time - dependent microstructure and mechanical properties of nanostructured Al-Si alloy

Efeoğlu, H. \& Turut, A., The current-voltage characteristics of the Au/MBE n-GaAs Schottky diodes in a wide temperature range

Ekuma, E. C., see Malozovsky, Y. El Haj Hassan, F., see Nemiri, O.

Elahi, S. M., see Taghavi Mendi, $\mathrm{R}$.

Elban, W. L., see Armstrong, R. W.

Elia, V., see Yinnon, T. A.

Enayati, M. H., see Azadehranjbar, S

Eskil, M. \& Kanca, E., Phase evolution in $\mathrm{Fe}-\mathrm{Mn}-\mathrm{Si}$ shape memory alloys due to forging speed

Fa, K. S. \& Wang, K. G., Integrodifferential equations associated with continuous-time random walk

Fafone, V., see Bassan, M.

Faizabadi, E., see Sattari, F.

Fan, J. D. \& Malozovsky, Y. M., An explanation of superfluidity of liquid ${ }^{3} \mathrm{He}$

Fan, J. D. \& Malozovsky, Y. M., Pauli exclusion principle
B27 (2013) 1350065

B27 (2013) 1350178 B27 (2013) 1361010

B27 (2013) 1362038 B27 (2013) 1362038

B27 (2013) 1345014

B27 (2013) 1345015 B27 (2013) 1345036 B27 (2013) 1350114

B27 (2013) 1350036

B27 (2013) 1350088

B27 (2013) 1362013

B27 (2013) 1350166

B27 (2013) 1350096

B27 (2013) 1330004

B27 (2013) 1350005

B27 (2013) 1350102

B27 (2013) 1350182

B27 (2013) 1330006

B27 (2013) 1350119

B27 (2013) 1350024

B27 (2013) 1362034

B27 (2013) 1362024
Fan, J. D. \& Malozovsky, Y. M., Sign reversal of Coulom interaction between two quasiparticles in momentum space

Fan, Y., see Li, M.

Fang, J. H., see Min, Y.

Fattorini, G., see Albertini, G.

Fazio, R., see Silvi, P.

Felder, B., see Deng, Z.

Feng, M., see Zang, C.-L.

Feng, M.-K., see Wang, X.-Y.

Feng, S. M., see Zhu, J. L.

Ferraz, Á., see Prudêncio, T.

Ferreira, A., see Bludov, Y. V.

Flores, Y., see Stashans, A.

Foggetta, L. G., see Bassan, M.

Frank, T. D., Kim, S. \& Dotov,

D. G., Canonical-dissipative nonequilibrium energy distributions: parameter estimation via implicit moment method, implementation and application

Franklin, L., see Malozovsky, Y.

Frasca, M., Localization in a strongly disordered system: A perturbation approach

Fu, S., see Luo, S.

Fu, X. L., Xing, Q. K., Peng, Z. J., Wang, C. B., Fu, Z. Q., Qi, L. H. \& Miao, H. Z., Microstructural and electromagnetic properties of $M n-Z n$ ferrites with low melting-point nonmagnetic $\mathrm{Sb}^{3+}$ ions

Fu, Z. Q., see Fu, X. L.

Fukuhara, M. \& Umemori, Y., Electronic transport behaviors due to charge density waves in $\mathrm{Ni}-\mathrm{Nb}-\mathrm{Zr}-\mathrm{H}$ glassy alloys

Gahlot, A. P. S., see Goswami, P.

Gandikota, R., see Mukhopadhyay, $\mathrm{S}$.

Gao, X., see Wang, X.

Gao, Y.-T., see Xu, X.-G.

Garcia-Barriocanal, J., see Santamaria, J.

Gaur, N. K., see Thakur, R.

Gautam, S., Generation of vortex dipoles in superfluid Fermi gas in BCS limit

Geraci, A. A., see Yin, Z.-Q.

Ghemid, S., see Nemiri, O.

Ghirardi, G. C. \& Romano, R., Classical, quantum and superquantum correlations
B27 (2013) 1362035

B27 (2013) 1350146

B27 (2013) 1350081

B27 (2013) 1350124

B27 (2013) 1345029

B27 (2013) 1362026

B27 (2013) 1350176

B27 (2013) 1350044

B27 (2013) 1362023

B27 (2013) 1350093

B27 (2013) 1341001

B27 (2013) 1350141

B27 (2013) 1350119

B27 (2013) 1350156 B27 (2013) 1362013

B27 (2013) 1350051 B27 (2013) 1345026

B27 (2013) 1350003

B27 (2013) 1350003

B27 (2013) 1350164 B27 (2013) 1330008

B27 (2013) 1350098

B27 (2013) 1350033 B27 (2013) 1250124

B27 (2013) 1330013 B27 (2013) 1350054

B27 (2013) 1350041

B27 (2013) 1330018 B27 (2013) 1350166

B27 (2013) 1345011 
Gholipur, R., see Bahari, A.

Ghosh, S., see Agrawal (Garg), N.

Ghulam Abbas, S. M., see Alay-eAbbas, S. M.

Giorgi, G. L. \& Busch, T., Genuine correlations in finite-size spin systems

Giovannetti, V., see Silvi, P.

Girolami, D., Vasile, R. \& Adesso, G., Theoretical insights on measuring quantum correlations

Goldbart, P. M., see Lu, B.-S.

Gong, M., see Cheng, Y.

Gong, P., Yao, K. \& Ding, H., Centimeter-sized Ti-based quaternary bulk metallic glass prepared by water quenching

González-Gaxiola, O., see Santiago, J. A.

Goswami, P., Gahlot, A. P. S. \& Singh, P., On $d+i d$ density wave and superconducting orderings in hole-doped cuprates

Graças, M., see Trindade, M. A. S. Grigorov, K. G., see Caetano, R.

Gu, M., see Modi, K.

Gu, S.-J., Yu, W.-C. \& Lin, H.Q., A spin chain with spiral orders: perspectives of quantum information and mechanical response

Guan, R.-H., see Cui, Y.-M.

Gui, Z., Wu, X. \& Chen, Y., Global synchronization of multi-scroll saturated chaotic systems via single-state linear feedback control

Gulácsi, Z., Exact ground states of correlated electrons on pentagon chains

Guo, C. Q., Zhang, C. Y., Hao, S. J., Jin, W. T. \& Zhang, H., Fixed triangle in $\mathrm{Bi}_{2-x} \mathrm{~Pb}_{x} \mathrm{Sr}_{2} \mathrm{CaCu}_{2} \mathrm{O}_{8+y}$ and $\mathrm{Bi}_{2-x} \mathrm{~Pb}_{x} \mathrm{Sr}_{2} \mathrm{Ca}_{2} \mathrm{Cu}_{3} \mathrm{O}_{10+y}$ systems

Guo, H. \& Shen, S.-Q., Effect of interaction in one-dimensional topological insulator

Guo, H., Chien, C.-C., He, Y. \& Levin, K., Fundamental constraints on linear response theories of fermi superfluids above and below $T_{c}$
B27 (2013) 1350153

B27 (2013) 1341003

B27 (2013) 1350100

B27 (2013) 1345034

B27 (2013) 1345029

B27 (2013) 1345020

B27 (2013) 1330012

B27 (2013) 1350130

B27 (2013) 1350087

B27 (2013) 1350043

B27 (2013) 1330008

B27 (2013) 1350133

B27 (2013) 1350089

B27 (2013) 1345027

B27 (2013) 1350106 B27 (2013) 1361006

B27 (2013) 1350007

B27 (2013) 1330009

B27 (2013) 1362015

B27 (2013) 1361001
Guo, M. \& Du, J., Electronic and optical properties of $\mathrm{C}-\mathrm{N}$ codoped $\mathrm{TiO}_{2}$ : a first-principles $G G A+U$ investigation

Guo, R., see Tan, L.

Guo, X., see Lu, X.

Guo, Y., Measurement-induced nonlocality over two-sided projective measurements

Haindl, S., Kidszun, M., Onken, F., Mietke, A. \& Thersleff, T., Lessons from oxypnictide thin films

Haldar, S. K. \& Chakrabarti, B., Dynamical features of Shannon information entropy of bosonic cloud in a tight trap

Hamilton, A. R., see Neilson, D.

Han, W., see Li, X.

Hang, C., see Du, Q.

Hanif, S., see Alay-e-Abbas, S. M. Hao, Q.-H., Li, Y. D., Kong, X.-S. \& Liu, C. S., Ab initio molecular dynamics simulations on local structure and electronic properties in liquid Sb from 913 K to $1193 \mathrm{~K}$

Hao, Q.-H., You, Y.-W., Kong, X.S. \& Liu, C. S., Ab initio molecular dynamics simulations on local structure and electronic properties in liquid $M g_{x} B i_{1-x}$ alloys

Hao, S. J., see Guo, C. Q.

Hara, S., see Deng, Z.

Hardy, L., Are quantum states real?

Hari Dass, N. D., Qureshi, T. \& Sheel, A., Minimum uncertainty and entanglement

Hayoune, A., A calorimetric study of the precipitation hardening mechanisms in an $\mathrm{Al}-\mathrm{Cu}-\mathrm{Mg}-$ Si alloy

$\mathrm{He}, \mathrm{J}$. , see Wu, C.

He, L., see Lu, X

He, M., see Tao, H. L.

$\mathrm{He}, \mathrm{P} .$, State-of-the-art investigations on statistical mechanics for self-gravitating systems

He, W., see Lai, F.

He, Y., see Guo, H.

Herrmann, R., Infrared spectroscopy of diatomic molecules - a fractional calculus ap-
B27 (2013) 1350123

B27 (2013) 1362017

B27 (2013) 1250212

B27 (2013) 1350067

B27 (2013) 1330001

B27 (2013) 1350048

B27 (2013) 1347004

B27 (2013) 1362021

B27 (2013) 1350065

B27 (2013) 1350100

B27 (2013) 1350012

B27 (2013) 1350011

B27 (2013) 1362015

B27 (2013) 1362026

B27 (2013) 1345012

B27 (2013) 1350068

B27 (2013) 1350084

B27 (2013) 1350165

B27 (2013) 1250212

B27 (2013) 1350078

B27 (2013) 1361011

B27 (2013) 1350127

B27 (2013) 1330010 
proach

Ho, C.-L., see Chou, C.-I.

Ho, Y. K., see Chen, Z.

Horng, L., see Young, S.-L.

Horodecki, M. \& Oppenheim, J., (Quantumness in the context of) resource theories

Hoyer, Y. D., see Caetano, R.

Hu, C. G., see Zhang, C. L.

Hu, F. M., see Sun, J. H.

Hu, K., see Tang, B.

Hu, P., see Tong, M.-W.

Hu, Y.-F., see Tang, C.-M.

Hu, Z.-W., see Wang, X.-Y.

Huang, G., see Du, Q.

Huang, M., see Li, T.-H.

Huang, S. J., see Xiao, T. T.

Huang, S., see Zhang, C.-H.

Huang, Y. L., see Wang, L. G.

Huang, Y., see Duan, H.

Huang, Y., see Liu, H.

Huang, Y.-L., see Yang, M.

Huang, Y.-N., see Zhou, H.-W.

Hudak, M. \& Hudak, O., Dynamic susceptibility in a paraelectric phase in a small particle, boundary effects

Hudak, O., see Hudak, M.

Husnain, G., see Shah, A.

Hussain, I., see Alay-e-Abbas, S. M.

Im, C., see Kim, S.

Ishiguri, S., Observation of new superconductivity and performance improvement by employing cyclotron of electron pairs

Ismail, R., see Kang, E. S.

Izumi, M., see Deng, Z.

Jang, H.-K., Lee, Y.-G., Shin, J.H. \& Kim, C.-G., Electromagnetic wave absorbing technique using periodic patterns for low $R C S$ patch array antenna

Jauregui, L. A., see Chung, T. F.

Javan, M. B., Electronic and optical properties of nitrogen doped $\mathrm{SiC}$ nanocrystals: First principles study

Jeon, J. E., see Kim, N.-H.

Ji, A. M., see Mao, L.-F.

Ji, G.-F., see Cheng, Y.

Ji, Y. H. \& Liu, Y. M., Study on quantum correlations for circuit QED states
B27 (2013) 1350019
B27 (2013) 1350135
B27 (2013) 1393001
B27 (2013) 1362006

B27 (2013) 1345019
B27 (2013) 1350089
B27 (2013) 1362010
B27 (2013) 1362039
B27 (2013) 1350139
B27 (2013) 1362008
B27 (2013) 1350040
B27 (2013) 1350195
B27 (2013) 1350065
B27 (2013) 1350148
B27 (2013) 1362040
B27 (2013) 1350147
B27 (2013) 1361005
B27 (2013) 1350178
B27 (2013) 1350174
B27 (2013) 1350006

B27 (2013) 1350080

B27 (2013) 1350015

B27 (2013) 1350015

B27 (2013) 1350020

B27 (2013) 1350100

B27 (2013) 1350010

B27 (2013) 1350045

B27 (2013) 1350077

B27 (2013) 1362026

B27 (2013) 1350094

B27 (2013) 1341002

B27 (2013) 1350053

B27 (2013) 1350030

B27 (2013) 1350172

B27 (2013) 1350130

B27 (2013) 1350056
Jia, D.-L., see Liu, Q.

Jiang, C., see Lai, F.

Jiang, J., Li, K. C., Zhao, L. F., Ma, J. Q., Zhang, Y. \& Zhao, Y., Vertical vibration characteristics of a high-temperature superconducting maglev vehicle system

Jiang, S.-H., see Wang, X.-Y.

Jin, C. Q., see Li, X.

Jin, C. Q., see Zhu, J. L.

Jin, W. T., see Guo, C. Q.

Jothiramalingam, R., see Nayak, P. K.

Kaatuzian, H., see Davoodi, H.

Kaatuzian, H., see Davoodi, H.

Kadri, M. T., see Ouchene, S.

Kajimoto, R., see Okuda, T.

Kanca, E., see Eskil, M.

Kang, E. S., Ahmadi, M. T., Anwar, S. \& Ismail, R., Energy quantization on the current-voltage characteristic of nanoscale two-dimensional Mosfet

Kao, M. C., see Chen, H. Z.

Kao, M.-C., see Young, S.-L.

Kapitanchuk, O. L., see Teslenko, V. I.

Kapor, D., see Vujinović, M.

Kar, M., see Mohanty, S.

Karimzadeh, F., see Azadehranjbar, S.

Kawarada, H., see Nishizaki, T.

Khachai, H., see Semari, F.

Khanata, R., see Ali, R.

Khenata, R., see Semari, F.

Khodel, V. A., see Clark, J. W.

Khosravi, E., see Tan, L.

Khusayfan, N. M., AC conductivity and dielectric properties of pulsed laser deposited CdS thin films

Kidszun, M., see Haindl, S.

Kim, A., see Cattani, F.

Kim, C.-G., see Jang, H.-K.

Kim, K., Rathnayaka, K. D. D., Lyuksyutov, I. F. \& Naugle, D. G., Transport properties of a superconducting film with magnetic nanostripes

Kim, K., Rathnayaka, K. K. D., Lyuksyutov, I. F. \& Naugle, D. G., Superconducting film with an array of magnetic nanos-
B27 (2013) 1350014 B27 (2013) 1350126

B27 (2013) 1362022

B27 (2013) 1350197

B27 (2013) 1362021

B27 (2013) 1362023

B27 (2013) 1362015

B27 (2013) 1250213

B27 (2013) 1350097

B27 (2013) 1392004

B27 (2013) 1350142

B27 (2013) 1330002

B27 (2013) 1350182

B27 (2013) 1350077

B27 (2013) 1362003

B27 (2013) 1362006

B27 (2013) 1350169

B27 (2013) 1350071

B27 (2013) 1350025

B27 (2013) 1350102

B27 (2013) 1362014

B27 (2013) 1350082

B27 (2013) 1350170

B27 (2013) 1350082

B27 (2013) 1347005

B27 (2013) 1362017

B27 (2013) 1350140

B27 (2013) 1330001

B27 (2013) 1330003

B27 (2013) 1350094

B27 (2013) 1362025 
tripes

Kim, K., see Lee, H.

Kim, K., see Ozmetin, A. E.

Kim, N.-H., Jeon, J. E., Cho, G.B., Lee, W.-S. \& Choi, D.-Y., Amorphous copper diselenide thin films doped with gallium and indium by laser-induced doping

Kim, S., Yeo, J. \& Im, C., Transit time distribution and mobility in Monte Carlo simulations of the Gaussian disorder model

Kim, S., see Frank, T. D.

Klyuev, A. V., Cumulant analysis of detection of random process using a Schottky diode with $\delta$ doping

Kobayashi, N., see Nishizaki, T.

Kofané, T. C., see Saha, M.

Kokoreva, M. A., see Pyataev, M. A.

Kolesnichenko, Y. A., see Belevtsev, B. I.

Kong, Q., see Chen, Z.

Kong, X.-S., see Hao, Q.-H.

Kong, X.-S., see Hao, Q.-H.

Kou, S.-P., see Zang, C.-L.

Kou, S.-P., see Zhang, X.-H.

Kteyan, A., see Vardanyan, A.

Kumar, R., see Bhalla, V.

Kung, C.-Y., see Young, S.-L.

Kurt, H., All-dielectric periodic media engineered for slow light studies

Kuzemsky, A. L., Unconventional and exotic magnetism in carbon-based structures and related materials

La, P., see Lu, X.

Lai, F., Zhang, Y.-P., Xia, H.-P., Wang, J.-H. \& He, W., Optical characterization of $\mathrm{Tb}^{3+}$. doped $\mathrm{Gd}_{2} \mathrm{O}_{3}$ based scintillating glasses

Lai, F., Zhang, Y.-P., Xia, H.-P., Wang, J.-H. \& Jiang, C., Spectroscopic properties of $\mathrm{Ce}^{3+}$-doped oxide glasses with high $\mathrm{Gd}_{2} \mathrm{O}_{3}$ concentrations

Landínez Téllez, D. A., see Rendón Ramírez, J. M.

Lee, H., Kim, K., Rathnayaka, K. K. D., Lyuksyutov, I. F. \& Naugle, D. G., Superconduct-
B27 (2013) 1362020

B27 (2013) 1362005

B27 (2013) 1362016

B27 (2013) 1350030

B27 (2013) 1350010

B27 (2013) 1350156

B27 (2013) 1350049

B27 (2013) 1362014

B27 (2013) 1350143

B27 (2013) 1350103

B27 (2013) 1350026

B27 (2013) 1393001

B27 (2013) 1350012

B27 (2013) 1350011

B27 (2013) 1350176

B27 (2013) 1250214

B27 (2013) 1250206

B27 (2013) 1350116

B27 (2013) 1362006

B27 (2013) 1330020

B27 (2013) 1330007

B27 (2013) 1250212

B27 (2013) 1350127

B27 (2013) 1350126

B27 (2013) 1350171 ing film with embedded cobalt nanorods

B27 (2013) 1362005

Lee, S.-H., Flock foraging efficiency in relation to food sensing ability and distribution: A simulation study

Lee, W.-C., Lv, W. \& Arham, H. Z., Elementary excitations due to orbital degrees of freedom in iron-based superconductors

Lee, W.-S., see Kim, N.-H.

Lee, Y.-G., see Jang, H.-K.

Lei, M., Pan, X., Yang, X., Wang, W., Yang, F. \& Zhao, Y., Preparation of $\mathrm{Sm}_{0.2} \mathrm{Ce}_{0.8} \mathrm{O}_{1.9-x}$ buffer by reel-to-reel slot-die coating

Lei, M., Yang, X. \& Zhao, Y., Fabrication of $\mathrm{SmBiO}_{3} / \mathrm{NiO}$ double buffer layer for coated conductors

Lei, Q., see Wang, J.

Leon, C., see Santamaria, J.

Levin, K., see Guo, H.

Li, B. \& Lin, D.-H., Numerical study of magnetic penetration depth in $\mathrm{MgB}_{2}$

$\mathrm{Li}, \mathrm{B}$., see $\mathrm{Wu}, \mathrm{C}$.

Li, C.-F., see Xu, J.-S.

Li, D. P., see Wang, H. F.

Li, D.-J., see Tang, B.

Li, F. J., Thermoelectricity of $\mathrm{Ca}_{2 .} \mathrm{Ce}_{0.1} \mathrm{Co}_{4} \mathrm{O}_{9+\delta} \quad$ based nanocomposites with $\mathrm{Cu}_{2} \mathrm{O}$ nanoinclusion

Li, F. \& Ma, J., Selection of spiral wave in the coupled network under Gaussian colored noise

Li, J.-C., see Wang, X.-X.

Li, J.-G., see Liu, B.-Q.

Li, K. C., see Jiang, J.

Li, L. T., see Zhu, J. L.

Li, L. \& Yu, B., Chaos in an adaptive network with rewiring

Li, M., Fan, Y., Wu, J. \& Di, Z., Phase transitions in Ising model induced by weight redistribution on weighted regular networks

Li, P., Zhao, Q. \& Wang, H., A weighted local-world evolving network model based on the edge weights preferential selection

Li, P.-C., see Lin, L.
B27 (2013) 1350146

B27 (2013) 1350145

B27 (2013) 1330014 B27 (2013) 1350030 B27 (2013) 1350094

B27 (2013) 1362019

B27 (2013) 1362018 B27 (2013) 1350132 B27 (2013) 1330013 B27 (2013) 1330010

B27 (2013) 1362027 B27 (2013) 1350165 B27 (2013) 1345054 B27 (2013) 1362029 B27 (2013) 1350139

B27 (2013) 1350108

B27 (2013) 1350115 B27 (2013) 1350050 B27 (2013) 1345055 B27 (2013) 1362022 B27 (2013) 1362023

B27 (2013) 1350104

B27 (2013) 1350039 B27 (2013) 1350180 
Li, Q., see Chen, T.-P.

Li, Q., see Tao, H. L.

Li, R.-Z., see Zhang, C.-H.

Li, S., Liu, Y., Ren, Z., Zhang, X. \& Liu, G., Electronic structure and half-metallicity of Heusler alloy $\mathrm{Mn}_{2} \mathrm{RhAl}$

Li, T., see Yin, Z.-Q.

Li, T.-H., Huang, M., Yang, J.-J., Lu, J. \& Cao, H.-L., A simplified parameter design method for transformation optics-based metamaterial innovative cloak

Li, X. D., see Li, X.

Li, X., Liu, Q. Q., Han, W., Liu, Y., Li, X. D., Li, Y. C., Liu, J. \& Jin, C. Q., Synthesis and structural stability of $\mathrm{BiRhO}_{3}$ at high pressure

Li, X., see Yang, P.

Li, Y. C., see Li, X.

Li, Y. D., see Hao, Q.-H.

Li, Y. Z., see Mao, L.-F.

Li, Y.-P., see Wang, X.-X.

Liang, B.-L., Wang, J.-S., Meng, X.-G. \& Yang, Q.-Y., Decoherence dynamics of a flux qubit respectively coupled to a boson bath and a spin bath

Liang, S.-D., see Wang, X.

Liao, B., see Chen, Y.-M.

Liao, H., see Yang, Q.

Liao, R.-J., see Wang, Y.-Y.

Ligi, C., see Bassan, M.

Lin, C.-C., see Young, S.-L.

Lin, C.-H., see Ou, C.-J.

Lin, C.-H., see Young, S.-L.

Lin, D., see Nian, F.

Lin, D.-H., see Li, B.

Lin, H. Q., see Sun, J. H.

Lin, H. \& Yau, S.-T., On exotic sphere fibrations, topological phases, and edge states in physical systems

Lin, H.-Q., see Gu, S.-J.

Lin, L., Li, P.-C. \& Tseng, H.C., A systematic study of nonlocally coupled oscillators and Chimera states

Lin, M.-M., see Yang, Y.

Lin, T.-T., see Young, S.-L.

Lin, Z. P., see Duan, H.

Lisak, M., see Cattani, F.

Liu, A.-P., Sun, X.-N. \& Yao, $\mathrm{X} . \mathrm{X}$., The simulation analysis
B27 (2013) 1362009

B27 (2013) 1350078

B27 (2013) 1350147

B27 (2013) 1350161

B27 (2013) 1330018

B27 (2013) 1350148

B27 (2013) 1362021

B27 (2013) 1362021

B27 (2013) 1350150

B27 (2013) 1362021

B27 (2013) 1350012

B27 (2013) 1350172

B27 (2013) 1350050

B27 (2013) 1350134

B27 (2013) 1350001

B27 (2013) 1350095

B27 (2013) 1350138

B27 (2013) 1350183

B27 (2013) 1350119

B27 (2013) 1362006

B27 (2013) 1362028

B27 (2013) 1362006

B27 (2013) 1350112

B27 (2013) 1362027

B27 (2013) 1362039

B27 (2013) 1350107

B27 (2013) 1350106

B27 (2013) 1350180

B27 (2013) 1361010

B27 (2013) 1362006

B27 (2013) 1350178

B27 (2013) 1330003 of nano-ZnO piezoelectric film based on SPM micro-cantilever

Liu, A.-P., see Sun, X.-S.

Liu, B.-Q., Shao, B., Li, J.-G. \& Zou, J., System-spin environment dynamics of quantum discord

Liu, C. S., see Hao, Q.-H.

Liu, C. S., see Hao, Q.-H.

Liu, G., Wu, M. S., Ouyang, C. Y. \& Xu, B., Structural and electronic evolution from $\mathrm{SiC}$ sheet to silicene

Liu, G., see Li, S.

Liu, H., Wang, H., Cao, M., Tan, W., Shi, Y., Chen, Y. \& Huang, Y., Effect of heat treatment on microstructure and magnetostrictive property of meltspun $\mathrm{Fe}_{85} \mathrm{Ga}_{15}$ ribbons

Liu, J., Tan, S.-B. \& Dong, H.-N., Stable structures, electric and magnetic properties of nanoparticles $\mathrm{CO}_{n}(n=1-6)$ clusters: First-principles calculations

Liu, J., see Li, X.

Liu, L. H., see Wang, H. F.

Liu, Q. Q., see Li, X.

Liu, Q. Q., see Zhu, J. L.

Liu, Q., Shen, S.-Y. \& Wang, Z.$\mathrm{H}$., The rational solutions to a generalized Riccati equation and their application

Liu, Q., Wang, Z.-H. \& Jia, D.L., A multiple Riccati equations rational-exponent method and its application to WhithamBroer-Kaup equation

Liu, R.-X., see Zhen, H.-L.

Liu, X.-R., see Xie, G.-Y.

Liu, Y. M., see Ji, Y. H.

Liu, Y., see Li, S.

Liu, Y., see Li, X.

Liu, Y.-K. \& Yang, S.-J., Fractional windings of the spinor condensates on a ring

Liu, Z., see Bergholtz, E. J.

Lo Franco, R., Bellomo, B., Maniscalco, S. \& Compagno, G., Dynamics of quantum correlations in two-qubit systems within non-Markovian environments

Lomnitz, M., Villarreal, C. \& de
B27 (2013) 1362031

B27 (2013) 1362030

B27 (2013) 1345055

B27 (2013) 1350012

B27 (2013) 1350011

B27 (2013) 1350188

B27 (2013) 1350161

B27 (2013) 1350174

B27 (2013) 1362007

B27 (2013) 1362021

B27 (2013) 1362029

B27 (2013) 1362021

B27 (2013) 1362023

B27 (2013) 1350013

B27 (2013) 1350014

B27 (2013) 1350029

B27 (2013) 1362032

B27 (2013) 1350056

B27 (2013) 1350161

B27 (2013) 1362021

B27 (2013) 1350070

B27 (2013) 1330017

B27 (2013) 1345053 
Llano, M., BEC model of high$T_{c}$ superconductivity in layered cuprates

Lorenzo, S., see Apollaro, T. J. G.

Lu, B.-S., Ye, F., Xing, X. \& Goldbart, P. M., Statistical physics of isotropic-genesis nematic elastomers: I. structure and correlations at high temperatures

Lu, J., see Li, T.-H.

Lu, J., see Zhao, H.

Lu, Q.-B., Cosmic-ray-driven reaction and greenhouse effect of halogenated molecules: Culprits for atmospheric ozone depletion and global climate change

Lu, W., see Zhao, H.

Lu, X., La, P., Guo, X., Wei, Y., Nan, X. \& He, L., Electronic structures and optical properties of $2 \mathrm{Al}$ - and $2 \mathrm{Ca}$-doped $\beta$ $\mathrm{Si}_{3} N_{4}$ : a first-principles study

Luo, C., see Wang, X.-Y.

Luo, C., see Wang, X.-Y.

Luo, H.-G., see Zhong, Y.

Luo, J. M., Modeling for formation of conducting path in $\mathrm{Cu} / \mathrm{SiO}_{2} / \mathrm{Pt}$ memory devices: Based on soft breakdown mechanism

Luo, M.-X., see Peng, J.-Y.

Luo, S. \& Fu, S., Hierarchy of correlations via Lüders measurements

Lv, G., see Cui, Y.-M.

Lv, L., Zhang, M., Wei, Z., Yang, L., Yang, X. \& Zhao, Y., Magnetoresistance in topological insulator $\mathrm{Mn}_{x} B i_{2-x} \mathrm{Se}_{3}$ crystals

Lv, L., Zhang, M., Wei, Z., Yang, L., Yang, X. \& Zhao, Y., Magnetoresistance in topological insulator $\mathrm{Mn}_{x} \mathrm{Bi}_{2-x} \mathrm{Se}_{3}$ crystals

Lv, L., see Yang, L. Q.

Lv, M. \& Zhang, S.-C., Dielectric function, friedel oscillation and plasmons in Weyl semimetals

Lv, W., see Lee, W.-C.

Lyu, H. \& Choi, J. R., High inductance coil embedded on magnetic sensor chip for biomagnetic signal measurements

Lyuksyutov, I. F., see Kim, K.

Lyuksyutov, I. F., see Kim, K.
B27 (2013) 1347001

B27 (2013) 1345035

B27 (2013) 1330012 B27 (2013) 1350148 B27 (2013) 1350086

B27 (2013) 1350073 B27 (2013) 1350086

B27 (2013) 1250212 B27 (2013) 1350195 B27 (2013) 1350197 B27 (2013) 1361002

B27 (2013) 1350160 B27 (2013) 1350091

B27 (2013) 1345026 B27 (2013) 1361006

B27 (2013) 1392002

B27 (2013) 1362036

B27 (2013) 1362012

B27 (2013) 1350177 B27 (2013) 1330014

B27 (2013) 1350159 B27 (2013) 1362020 B27 (2013) 1362025
Lyuksyutov, I. F., see Lee, H.

Lyuksyutov, I. F., see Ozmetin, A. E.

Lyuksyutov, I. F., Controlling superconductivity with magnetic nanostructures

Ma, J. Q., see Jiang, J.

Ma, J., see Li, F.

Ma, R., Thermoelectric transport properties in trilayer graphene systems

Ma, Y., see Wang, X.-Y.

Ma, Y., see Wang, X.-Y.

Ma, Y., see Yang, Q.

Madhok, V. \& Datta, A., Quantum discord as a resource in quantum communication

Magpantay, J. A., Microscopic irreversibility and the $H$ theorem

Mahmood, A., see Shah, A.

Mahmoodi, N., see Azadehranjbar, S.

Majd, A. E., see Asadpour, S. H.

Majidiyan, M., see Taghavi Mendi, R.

Majumdar, P., see Tiwari, R.

Mali, P., see Vujinović, M.

Malik, F. B., see Bohr, H. G.

Malik, F. B., see Sarris, C. M.

Malik, G. P. \& de Llano, M., An alternative approach to multi-gap superconductivity

Malik, G. P. \& Varma, V. S., A comparative study of the experimental features of the BoseEinstein condensates of ${ }^{7} \mathrm{Li}$, ${ }^{23} \mathrm{Na},{ }^{41} \mathrm{~K},{ }^{85} \mathrm{Rb},{ }^{87} \mathrm{Rb}$ and ${ }^{133} \mathrm{Cs}$ via a linearly perturbed harmonic oscillator potential

Malozovsky, Y. M., see Fan, J. D.

Malozovsky, Y. M., see Fan, J. D.

Malozovsky, Y. M., see Fan, J. D.

Malozovsky, Y., Franklin, L., Ekuma, E. C., Zhao, G. L. \& Bagayoko, D., Ab-initio calculations of electronic properties of InP and GaP

Mamedov, T. A. \& de Llano, M., Uniform Coulomb field as origin of "Fermi Arcs" in an anisotropic Boson-Fermion gas mixture

Mancini, S., see Angelakis, D.

Maniscalco, S., see Lo Franco, R.

Mao, L.-F., Ji, A. M., Zhu, C. Y.,
B27 (2013) 1362013

B27 (2013) 1362005

B27 (2013) 1362016

B27 (2013) 1362004

B27 (2013) 1362022

B27 (2013) 1350115

B27 (2013) 1361004

B27 (2013) 1350196

B27 (2013) 1350201

B27 (2013) 1350138

B27 (2013) 1345041

B27 (2013) 1250205

B27 (2013) 1350020

B27 (2013) 1350102

B27 (2013) 1350004

B27 (2013) 1350096

B27 (2013) 1350018

B27 (2013) 1350071

B27 (2013) 1347007

B27 (2013) 1347006

B27 (2013) 1347008

B27 (2013) 1350042

B27 (2013) 1362024

B27 (2013) 1362034

B27 (2013) 1362035

B27 (2013) 1347002

B27 (2013) 1345037

B27 (2013) 1345053 
Wang, Z. O., Zhang, L. J., Li, Y. Z., Wang, S. D. \& Yan, Y., The kink effects in nano-GaAs devices due to multi-valley electron transport

March, N. H., Angilella, G. G. N. \& Pucci, R., Natural orbitals in relation to quantum information theory: From model light atoms through to emergent metallic properties

Marini, A., see Bassan, M.

Martins, R., see Trindade, M. A. S.

Matos, T., see Castellanos, E.

Mazzitelli, G., see Bassan, M.

Maćkowiak, J., Dressed Bosons and effective temperature II

Mehaney, A., see Aly, A. H.

Meng, X.-G., see Liang, B.-L.

Meng, X.-H., see Xu, X.-G.

Meradji, H., see Nemiri, O.

Metwally, N., Abdel-Aty, M. \& Obada, A.-S., Coherent and incoherent behaviors of qubits interacting with a spin-path particle

Metwally, N., Usefulness classes of traveling entangled channels in noninertial frames

Miao, B., On the structure of statistical field theory of polymers

Miao, H. Z., see Fu, X. L.

Mietke, A., see Haindl, S.

Mignani, R., see Albertini, G.

Miki, M., see Deng, Z.

Min, Y., Fang, J. H., Zhong, C. G., Dong, Z. C., Chen, C. P. \& Yao, $\mathrm{K}$. L., Localization of the energy states of lead inducing the effect of rectification and negative differential resistance predicted by first-principles study

Mo, Z.-W., see Peng, J.-Y.

Mo, Z.-W., see Peng, J.-Y.

Modestino, G., see Bassan, M.

Modi, K. \& Gu, M., Coherent and incoherent contents of correlations

Moeenfard, H., Darvishian, A. \& Ahmadian, M. T., Analytical modeling of the effects of electrostatic actuation and casimir force on the pull-in instability and static behavior of torsional
B27 (2013) 1350172

B27 (2013) 1330021 B27 (2013) 1350119

B27 (2013) 1350133

B27 (2013) 1350060

B27 (2013) 1350119

B27 (2013) 1350131

B27 (2013) 1350047

B27 (2013) 1350134

B27 (2013) 1250124

B27 (2013) 1350166

B27 (2013) 1350076

B27 (2013) 1350155

B27 (2013) 1361009

B27 (2013) 1350003

B27 (2013) 1330001

B27 (2013) 1350124

B27 (2013) 1362026

B27 (2013) 1350081

B27 (2013) 1350091

B27 (2013) 1350137

B27 (2013) 1350119

B27 (2013) 1345027 nano/micro actuators

Mohanty, S., Kar, M. \& Ravi, S., Ferromagnetism in mechanically milled pure $\mathrm{SnO}_{2}$

Moon, K., see Rhim, J.-W.

Moon, S. J., see Son, S.

Mukherjee, K., see Acharya, S.

Mukherjee, K., see Acharya, S.

Mukhopadhyay, S., Demircioglu, B., Gandikota, R. \& Chatterjee, A., Ground state energy of a two-level system interacting with phonons

Murtaza, G., see Ali, R.

Murtaza, G., see Semari, F.

Nag, T., Dutta A. \& Patra, A., Quenching dynamics and quantum information

Nagao, M., see Nishizaki, T.

Nan, X., see Lu, X.

Naugle, D. G., see Kim, K.

Naugle, D. G., see Kim, K.

Naugle, D. G., see Lee, H.

Naugle, D. G., see Ozmetin, A. E.

Nayak, P. K. \& Jothiramalingam, R., Synthesis and characterization of zinc ferrite by XRD, $V S M$ and electron spin resonance techniques

Neilson, D., Hamilton, A. R. \& Thakur, J. S., Quantum glass transition at finite temperature in two-dimensional electron layers

Nemiri, O., Ghemid, S., Chouahda, Z., Meradji, H. \& El Haj Hassan, F., Structural, electronic, thermodynamic and thermal properties of zincblende InP, InAs and their InAs ${ }_{x} P_{1-x}$ ternary alloys via first principles calculations

Neto, M. A., see Salmon, O. D. R.

Newell, D., see Chung, T. F.

Nian, F. \& Wang, X., Projective synchronization of two different dimensional nonlinear systems

Nian, F., Wang, X. \& Zheng, P., Projective synchronization in chaotic complex system with time delay

Nian, F., Wang, X., Lin, D. \& Niu, Y., High precision fast projective synchronization for chaotic
B27 (2013) 1350008

B27 (2013) 1350025

B27 (2013) 1362011

B27 (2013) 1350151

B27 (2013) 1350057

B27 (2013) 1392003

B27 (2013) 1350098

B27 (2013) 1350170

B27 (2013) 1350082

B27 (2013) 1345036

B27 (2013) 1362014

B27 (2013) 1250212

B27 (2013) 1362020

B27 (2013) 1362025

B27 (2013) 1362005

B27 (2013) 1362016

B27 (2013) 1250213

B27 (2013) 1347004

B27 (2013) 1350166

B27 (2013) 1350162

B27 (2013) 1341002

B27 (2013) 1350113

B27 (2013) 1350111 
systems with unknown parameters

Nishiyama, S., da Providência, J., Providência, C. \& Ohnishi, H., An attempt at a resonating mean-field theoretical description of thermal behavior of twogap superconductivity

Nishizaki, T., Sasaki, T., Kobayashi, N., Takano, Y., Nagao, M. \& Kawarada, H., Spatial variation of tunneling spectra in (111)-oriented films of boron-doped diamond probed by STM/STS

Niu, Y., see Nian, F.

Nonomura, Y., Numerical study on irreversible behavior of $\mathrm{THz}$ wave emission from intrinsic Josephson junctions

Noor, K. I. \& Ahmad, K., On higher order Bazilevic functions

Obada, A.-S., see Metwally, N.

Ohnishi, H., see Nishiyama, S.

Okawa, M., see Okuda, T.

Okuda, T., Kajimoto, R., Okawa, M. \& Saitoh, T., Effects of holedoping and disorder on the magnetic states of delafossite $\mathrm{CuCrO}_{2}$ having a spin-3/2 antiferromagnetic triangular sublattice

Olivares, S. \& Paris, M. G. A., The balance of quantum correlations for a class of feasible tripartite continuous variable states

Olorunsola, O., Dada, O. \& Wang, P., A spinning polarizer and spinning analyzer method for visualizing the isochromates in conoscopic interferometers

Omran, S. B., see Ali, R.

Onken, F., see Haindl, S.

Oppenheim, J., see Horodecki, M.

Osman, K. I., see Abd-Elnabi, S.

Osterloh, A., Entanglement and its multipartite extensions

Ou, C.-J., Lin, C.-H. \& Ou, C.-M., Novel approach for $t-J$ model with electron scatterings

Ou, C.-J., see Young, S.-L.

Ou, C.-M., see Ou, C.-J.

Ouahrani, T., see Semari, F.

\section{B27 (2013) 1350112}

B27 (2013) 1350079

B27 (2013) 1362014 B27 (2013) 1350112

B27 (2013) 1362033

B27 (2013) 1250203

B27 (2013) 1350076

B27 (2013) 1350079

B27 (2013) 1330002

B27 (2013) 1330002

B27 (2013) 1345024

B27 (2013) 1350175

B27 (2013) 1350170

B27 (2013) 1330001

B27 (2013) 1345019

B27 (2013) 1350037

B27 (2013) 1345018

B27 (2013) 1362028

B27 (2013) 1362006

B27 (2013) 1362028

B27 (2013) 1350082
Ouchene, S., Kadri, M. T., Baaouague, K. \& Belkhir, H., Structural, electronic and elastic properties of ternary alloy $\mathrm{Co}_{x} \mathrm{Ni}_{1-x} \mathrm{Si}_{2}$

Ourabah, K. \& Tribeche, M., Nonextensive statistical mechanics approach to the sommerfeld model for metallic elements

Ouyang, C. Y., see Liu, G.

Ozisik, H. B., Colakoglu, K. \& Deligoz, E., Thermal properties of the $X B_{2}(X=A g, A u)$ compounds: A first-principles study Ozisik, H. B., see Ateser, E.

Ozmetin, A. E., Yazici, E., Kim, K., Rathnayaka, K. D. D., Lyuksyutov, I. F. \& Naugle, D. G., Hysteresis of the phase diagram in the ferromagnetsuperconductor hybrids

B27 (2013) 1362016

Padilha, I. T., see Salmon, O. D. R.

Pahuja, A. \& Srivastava, S., DFT study of structural and electronic properties of endohedral complexes of group $V$ atoms with $C_{60}$

Pal, A. K., see Bose, I.

Pan, L. L., see Tao, H. L.

Pan, W., see Zhao, R.

Pan, X., see Lei, M.

Pang, X.-F., The mechanism and properties of electron transfer in the biological organism

Pantić, M., see Vujinović, M.

Paris, M. G. A., see Olivares, S.

Park, S.-J., Shima, H. \& Sato, M., Kaleidoscopic mode change in cross-sectional deformation of reinforced carbon nanotubes

Patra, A., see Nag, T.

Pavlov, P. A., see Vinogradov, V. E.

Peng, J.-Y., Bai, M.-Q. \& Mo, Z.-W., Remote information concentration by $W$ state

B27 (2013) 1350181

B27 (2013) 1350188

B27 (2013) 1350046

B27 (2013) 1350016

B27 (2013) 1350162

B27 (2013) 1350152

B27 (2013) 1345042

B27 (2013) 1350078

B27 (2013) 1350129

B27 (2013) 1362019

B27 (2013) 1350090

B27 (2013) 1350071

B27 (2013) 1345024

B27 (2013) 1350179

B27 (2013) 1345036

B27 (2013) 1350128

B27 (2013) 1350137

Peng, J.-Y., Luo, M.-X. \& Mo, Z.$\mathrm{W}$, Remote information concentration via four-particle cluster state and by positive operator-value measurement

Peng, Z. J., see Fu, X. L.

Perdew, J. P., see Tao, J.
B27 (2013) 1350091 B27 (2013) 1350003 B27 (2013) 1330011 
Peres, N. M. R., see Bludov, Y. V. Petrucci, A., see Albertini, G. Pizzella, G., see Bassan, M.

Plastina, F., see Apollaro, T. J. G. Pradhan, B., see Raj, B. K.

Proto, A. N., see Sarris, C. M.

Providência, C., see Nishiyama, S. Prudêncio, T. \& Ferraz, Á., Confinement and quantum anomaly in quasi-1D spinless fermion chains

Prudêncio, T., Confinement and quantum anomaly in quasi-1D spinless Fermion chains

Pruessner, G., The average avalanche size in the Manna model and other models of selforganized criticality

Prykarpatsky, Y. A., see Bogolubov Jr, N. N..

Pucci, R., see March, N. H.

Pyataev, M. A. \& Kokoreva, M. A., Spectral and transport properties of one-dimensional nanoring superlattice

Qi, F., see Wu, C.

Qi, L. H., see Fu, X. L.

Qian, P., see Wang, X.-X.

Qian, T., see Xu, X.

Qing, Z.-H., see Tong, M.-W.

Qu, S., see Ye, H.

Quintieri, L., see Bassan, M.

Qureshi, T., see Hari Dass, N. D.

Rabah, M., see Semari, F.

Rabhi, A., Brajczewska, M., Schuck, P., Da Providência, J. \& Bennaceur, R., The $1 D$ Heisenberg antiferromagnet model by the variation after projection method

Rached, D., see Semari, F.

Raina, K. K., see Singh, R.

Raj, B. K., Pradhan, B. \& Rout, G. C., Modified BCS gap equation for Jahn-Teller distorted high temperature superconductors

Rathnayaka, K. D. D., see Kim, K.

Rathnayaka, K. D. D., see Ozmetin, A. E.

Rathnayaka, K. K. D., see Kim, K. Rathnayaka, K. K. D., see Lee, H. Ravi, S., see Mohanty, S.

Reboiro, M., Civitarese, O. \& Tielas, D., Information entropy and spin-squeezing in atomic
B27 (2013) 1341001
B27 (2013) 1350124
B27 (2013) 1350119
B27 (2013) 1345035
B27 (2013) 1350059
B27 (2013) 1347006
B27 (2013) 1350079

B27 (2013) 1350093

B27 (2013) 1392005

B27 (2013) 1350009

B27 (2013) 1350002 B27 (2013) 1330021

B27 (2013) 1350103 B27 (2013) 1350165 B27 (2013) 1350003 B27 (2013) 1350050 B27 (2013) 1361008 B27 (2013) 1362008 B27 (2013) 1350144 B27 (2013) 1350119 B27 (2013) 1350068 B27 (2013) 1350082

B27 (2013) 1350058 B27 (2013) 1350082 B27 (2013) 1350038

B27 (2013) 1350059 B27 (2013) 1362025

B27 (2013) 1362016 B27 (2013) 1362020 B27 (2013) 1362005 B27 (2013) 1350025 three-level systems

Ren, Z., see Li, S.

Rendón Ramírez, J. M., Almanza, O. A. M., Cardona, R., Landínez Téllez, D. A. \& Roa-Rojas, J., Structural, magnetic and electronic properties of the $\mathrm{Sr}_{2} \mathrm{CoNbO}_{6}$ complex perovskite

Rhim, J.-W. \& Moon, K., Edge magnetism and quantum spin hall effect in zigzag graphene nanoribbon

Ribeiro, G. A. P., see Werlang, T.

Ridolfi, F., see Albertini, G.

Rigolin, G., see Werlang, T.

Roa-Rojas, J., see Rendón Ramírez, J. M.

Romano, R., see Ghirardi, G. C.

Ronga, F., see Bassan, M.

Rossignoli, R., see Canosa, N.

Rossini, D., see Silvi, P.

Rotili, A., see Albertini, G.

Rout, G. C., see Raj, B. K.

Ruzsinszky, A., see Tao, J.

Saha, A., Electron-electron interaction effects on transport through mesoscopic superconducting hybrid junctions

Saha, M. \& Kofané, T. C., Longrange interactions in the helicoidal DNA dynamics

Sahoo, B. K., Sahoo, S. K. \& Sahoo, S., The effect of spontaneous and piezoelectric polarization on thermal conductivity of $\operatorname{InN}$

Sahoo, M. \& Behera, D., Inhomogeneity induced conductivity fluctuation in $\mathrm{YBa}_{2} \mathrm{Cu}_{3} \mathrm{O}_{7-\delta} / \mathrm{BaTiO}_{3}-$ $\mathrm{CoFe}_{2} \mathrm{O}_{4}$ composite

Sahoo, S. K., see Sahoo, B. K.

Sahoo, S., see Sahoo, B. K.

Saitoh, T., see Okuda, T.

Sakamoto, S., see Tsuyuki, H.

Salmon, O. D. R., Crokidakis, N., Neto, M. A., Padilha, I. T., Viana, J. R. \& de Sousa, J. R., Qualitative aspects of the phase diagram of $J_{1}-J_{2}$ model on the cubic lattice

Santamaria, J., GarciaBarriocanal, J., Sefrioui, Z. \& Leon, C., Induced magnetism at
B27 (2013) 1350117 B27 (2013) 1350161

B27 (2013) 1350171
B27 (2013) 1362011
B27 (2013) 1345032
B27 (2013) 1350124
B27 (2013) 1345032
B27 (2013) 1350171
B27 (2013) 1345011
B27 (2013) 1350119
B27 (2013) 1345033
B27 (2013) 1345029
B27 (2013) 1350124
B27 (2013) 1350059
B27 (2013) 1330011

B27 (2013) 1330015

B27 (2013) 1350143

B27 (2013) 1350031

B27 (2013) 1350099

B27 (2013) 1350031

B27 (2013) 1350031

B27 (2013) 1330002

B27 (2013) 1350157

B27 (2013) 1350162 
Author Index

oxide interfaces

Santiago, J. A., Chacón-Acosta, G. \& González-Gaxiola, O., Elastic curves and surfaces under long-range forces: A geometric approach

Santoro, G. E., see Silvi, P.

Santos, L. C., see Trindade, M. A. S.

Sarandy, M. S., de Oliveira, T. R. \& Amico, L., Quantum discord in the ground state of spin chains

Saritaş, S., see Yurtseven, H.

Sarris, C. M., Proto, A. N. \& Malik, F. B., Gibbs entropy approach to the realization of qubits and qutrits

Sasaki, T., see Nishizaki, T.

Satarić, M. V., see Zdravković, S.

Sato, M., see Park, S.-J.

Sattari, F. \& Faizabadi, E., Band gap opening effect on the transport properties of bilayer graphene superlattice

Schmidt, H.-J., The general spin triangle

Schuck, P., see Rabhi, A.

Sefrioui, Z., see Santamaria, J.

Semari, F., Ouahrani, T., Khachai, H., Khenata, R., Rabah, M., Bouhemadou, A., Murtaza, G., Amin, B. \& Rached, D., Electronic band structure, optical, thermal and bonding properties of $\mathrm{XMg}_{2} \mathrm{O}_{4}(x=\mathrm{Si}, \mathrm{Ge})$ spinel compounds

Sereika, R., see Audzijonis, A.

Sergi, A. \& Zloshchastiev, K. G., Non-Hermitian quantum $d y$ namics of a two-level system and models of dissipative environments

Serra, R. M., see da Silva Jr, M. A.

Shah, A., Husnain, G., Ahmad, I. \& Mahmood, A., Cu ions irradiation impact on structural and optical properties of GaN thin film

Shao, B., see Liu, B.-Q.

Shao, C.-X., Dou, H.-L., Yang, R.X. \& Wang, B.-H., Zero nodes effect: Valid link prediction in sparse networks

Sharif, M., see Alay-e-Abbas, S.
B27 (2013) 1330013

B27 (2013) 1350043

B27 (2013) 1345029

B27 (2013) 1350133

B27 (2013) 1345030

B27 (2013) 1350035

B27 (2013) 1347006

B27 (2013) 1362014

B27 (2013) 1350184

B27 (2013) 1350179

B27 (2013) 1350024

B27 (2013) 1350064

B27 (2013) 1350058

B27 (2013) 1330013

B27 (2013) 1350082 B27 (2013) 1350122

B27 (2013) 1350163 B27 (2013) 1345013

B27 (2013) 1350020 B27 (2013) 1345055

B27 (2013) 1350052
M.

Sharma, M., see Agrawal (Garg), N.

Shaukat, A., see Alay-e-Abbas, S. M.

Sheel, A., see Hari Dass, N. D.

Shen, H. Q. \& Wang, Y. G., Tunable resonant frequency of $\mathrm{FeCo} / \mathrm{PZT} / \mathrm{Fe}$ Co cylinders

Shen, J., see Zhang, C.-H.

Shen, S.-Q., see Guo, H.

Shen, S.-Y., see Liu, Q.

Shen, T., see Chung, T. F.

Shi, C., see Chen, $\mathrm{H}$.

Shi, J.-J., see Zhang, M.

Shi, Y., see Liu, H.

Shih, Y.-T., see Young, S.-L.

Shiibashi, T., see Tsuyuki, H.

Shima, H., see Park, S.-J.

Shin, J.-H., see Jang, H.-K.

Shripal, Dwivedi, S., Singh, R. \& Tandon, R. P., Effects of copper doping on dielectric and A.C. conductivity in layered sodium tri-titanate ceramic

Silva Filho, L. M., see Trindade, M. A. S.

Silvi, P., Rossini, D., Fazio, R., Santoro, G. E. \& Giovannetti, V., Matrix product state representation for Slater determinants and configuration interaction states

Sima, W.-X., see Yang, M.

Singh, D., see Bhalla, V.

Singh, P., see Goswami, P.

Singh, R. \& Raina, K. K., Electrooptical studies in a ferroelectric liquid crystal mixture

Singh, R., see Shripal

Sismanoglu, B. N., see Caetano, R.

Siurakshina, L., see Yushankhai, $\mathrm{V}$.

Soleimani, H. R., see Asadpour, S. $\mathrm{H}$.

Soller, H., FCS of superconducting tunnel junctions in nonequilibrium

Soltani, A., see Asadpour, S. H.

Son, S. \& Moon, S. J., Unstable electromagnetic modes in strongly magnetized plasmas

Song, B., see Tao, H. L.

Song, X. K., see Xu, S.
B27 (2013) 1350100

B27 (2013) 1341003

B27 (2013) 1350100 B27 (2013) 1350068

B27 (2013) 1350173

B27 (2013) 1350147

B27 (2013) 1361001

B27 (2013) 1350013

B27 (2013) 1341002

B27 (2013) 1350149

B27 (2013) 1350186

B27 (2013) 1350174

B27 (2013) 1362006

B27 (2013) 1350157

B27 (2013) 1350179

B27 (2013) 1350094

B27 (2013) 1350114

B27 (2013) 1350133

B27 (2013) 1345029

B27 (2013) 1350006

B27 (2013) 1350116

B27 (2013) 1330008

B27 (2013) 1350038

B27 (2013) 1350114

B27 (2013) 1350089

B27 (2013) 1350185

B27 (2013) 1350004

B27 (2013) 1350072

B27 (2013) 1350004

B27 (2013) 1350151

B27 (2013) 1350078

B27 (2013) 1350074 
Song, X.-K., see Wu, T.

Srivastava, S., see Pahuja, A.

Stashans, A. \& Flores, Y., Modelling of neutral vacancies in forsterite mineral

Sun, J. H., Hu, F. M., Tang, H. K. \& Lin, H. Q., Magnetic impurity in the vicinity of a vacancy in bilayer graphene

Sun, J.-X., see Zhou, S.

Sun, X.-N., see Liu, A.-P.

Sun, X.-S., Liu, A.-P. \& Yao, X.$\mathrm{X}$., The application for PZT thin film in the tapping mode SPM cantilever

Taghavi Mendi, R., Majidiyan, M., Boochani, A., Elahi, S. M., Bakhshayeshi, A. \& Beryani Nezafat, N., Study of V substitution effect on structural and electronic and magnetic properties of $Z n_{1-x} V_{x} O 0 \leq x \leq 0.5$ by first principles calculations

Takano, Y., see Nishizaki, T.

Tan, L., Guo, R., Yang, S., Khosravi, E., Zhao, G.-L. \& Bagayoko, D., Electronic structure of $\mathrm{K}_{0.8} \mathrm{Fe}_{2} \mathrm{Se}_{2}$ from density functional theory GW method simulation

Tan, S.-B., see Liu, J.

Tan, W., see Liu, H.

Tan, Y., see Wang, X.-Y.

Tandon, R. P., see Shripal

Tang, B., Li, D.-J., Hu, K. \& Tang, Y., Intrinsic localized modes in quantum ferromagnetic IsingHeisenberg chains with singleion uniaxial anisotropy

Tang, C.-M., Chen, X.-X., Wang, J., Hu, Y.-F. \& Wang, H.-Y., First-principle investigation of electric structures and thermodynamic properties for zirconium nitride under high pressure

Tang, H. K., see Sun, J. H.

Tang, J. L., see Xiao, T. T.

Tang, L., Xu, Z. \& Yang, Z., First-principles calculations of current-induced spin-transfer torques in magnetic domain walls

Tang, Y., see Tang, B.

Tang, Y., see Wang, H.
B27 (2013) 1350136 B27 (2013) 1350152

B27 (2013) 1350141

B27 (2013) 1362039

B27 (2013) 1350167

B27 (2013) 1362031

B27 (2013) 1362030

B27 (2013) 1350096

B27 (2013) 1362014

B27 (2013) 1362017

B27 (2013) 1362007

B27 (2013) 1350174

B27 (2013) 1350202

B27 (2013) 1350114

B27 (2013) 1350139

B27 (2013) 1350040 B27 (2013) 1362039 B27 (2013) 1362040

B27 (2013) 1350092

B27 (2013) 1350139 B27 (2013) 1350061
Tao, H. L., Zhang, Z. H., Pan, L. L., He, M., Song, B. \& Li, Q., Effects of oxygen vacancy on magnetic properties of cobalt-doped $\mathrm{ZnO}$ dilute magnetic semiconductors

Tao, J., Perdew, J. P. \& Ruzsinszky, A., Long-range van der Waals interaction

Tarasov, V. E., Review of some promising fractional physical models

Teslenko, V. I. \& Kapitanchuk, O. L., Theory of kinetics of multistep ligand-receptor assembly in dissipating and fluctuating environments

Thakur, J. S., see Neilson, D.

Thakur, R. K., see Thakur, R.

Thakur, R., Thakur, R. K. \& Gaur, N. K., Elastic and thermal properties of $\mathrm{Sr}_{1-x} \mathrm{Ca}_{x} \mathrm{RuO}_{3}$

Thapa, S. K. \& Adhikari, N. P., A molecular dynamics study of oxygen gas in water at different temperatures

Thersleff, T., see Haindl, S.

Tian, B., see Zhen, H.-L.

Tian, H. Y., see Wang, H. F.

Tian, M., see Wang, Y.-Y.

Tielas, D., see Reboiro, M.

Tipparaach, U., see Chen, T.-P.

Tiwari, R. \& Majumdar, P., Noncollinear magnetic order in the double perovskites: Double exchange on a geometrically frustrated lattice

Tomiya, M., see Tsuyuki, H.

Tong, M.-W., Hu, P., Qing, Z.-H., Zhang, Q. \& Chen, C., The performance characteristics of a new ceiling cooling panel

Tribeche, M., see Ourabah, K.

Trindade, M. A. S., Silva Filho, L. M., Santos, L. C., Graças, M., Martins, R. \& Vianna, J. D. M., Quantum information, thermofield dynamics and thermalized Bosonic oscillator

Tripathy, C., see Bhalla, V.

Tseng, H.-C., see Lin, L.

Tsuyuki, H., Shiibashi, T., Sakamoto, S. \& Tomiya, M., Effects of substitutional doping
B27 (2013) 1350078

B27 (2013) 1330011

B27 (2013) 1330005

B27 (2013) 1350169

B27 (2013) 1347004

B27 (2013) 1350054

B27 (2013) 1350054

B27 (2013) 1350023

B27 (2013) 1330001

B27 (2013) 1350029

B27 (2013) 1362029

B27 (2013) 1350183

B27 (2013) 1350117

B27 (2013) 1362009

B27 (2013) 1350018 B27 (2013) 1350157

B27 (2013) 1362008 B27 (2013) 1350181

B27 (2013) 1350133

B27 (2013) 1350116

B27 (2013) 1350180 
in electronic transport properties of carbon nanotubes

Tsuzuki, K., see Deng, Z.

Turut, A., see Efeoğlu, H.

Umemori, Y., see Fukuhara, M.

Valente, P., see Bassan, M.

Vardanyan, A. \& Kteyan, A., Stochastic dynamics of DC and AC driven dislocation kinks

Varma, V. S., see Malik, G. P.

Vasile, R., see Girolami, D.

Vasilevskiy, M. I., see Bludov, Y. $\mathrm{V}$.

Vedral, V., see Dorner, R.

Viana, J. R., see Salmon, O. D. R.

Vianna, J. D. M., see Trindade, M. A. S.

Villarreal, C., see Lomnitz, M.

Vinko, J. D., see Dufour, J. J.

Vinogradov, V. E. \& Pavlov, P. A., Cavitation strength of liquids with large molecules

Vujinović, M., Pantić, M., Kapor, D. \& Mali, P., Theoretical models for magnetic properties of iron pnictides

Walley, S. M., see Armstrong, R. W.

Wan, J., see Wu, C.

Wang, B.-H., see Shao, C.-X.

Wang, C. B., see Fu, X. L.

Wang, C. \& Cao, J. C., Study on small-signal impedance of single-walled zigzag carbon nanotubes in terahertz frequency regime

Wang, C., see Ye, H.

Wang, C.-J., Delays induce different switch in a stochastic single genetic regulation system with a positive autoregulatory feedback loop

Wang, C.-Y., Yang, W.-X. \& $\mathrm{Du}, \mathrm{H}$., Superiority of generalized bond-moving renormalization in studying phase transition of complex spin systems

Wang, D., Weerasinghe, J., Albarakati, A. \& Bellaiche, L., Terahertz dielectric response and coupled dynamics of ferroelectrics and multiferroics from effective Hamiltonian simulations
Wang, D., see Ye, H.

B27 (2013) 1350144

B27 (2013) 1362029

B27 (2013) 1350158

Wang, G.-R., see Yuan, G.-Y.

Wang, H. F., Zhang, Y. Z., Liu, L. H., Li, D. P., Wang, G. Y., Tian, H. Y. \& Zheng, D. N., Transport properties of $\mathrm{La}_{2-x 1} \mathrm{Sr}_{x 1} \mathrm{CuO}_{4+\delta} / \mathrm{La}_{2-x 2} \mathrm{Sr}_{x 2} \mathrm{CuO}_{4+\delta}$ multilayers grown by laser $a b$ lation

B27 (2013) 1362029

B27 (2013) 1350042

B27 (2013) 1345020

B27 (2013) 1341001

B27 (2013) 1345017

B27 (2013) 1350162

B27 (2013) 1350133

B27 (2013) 1347001

B27 (2013) 1362038

B27 (2013) 1350128

Wang, H., Cheng, X., Zhang, H. \& Tang, Y., Very high hydrogen storage capacity of al-adsorbed single-walled carbon nanotube (SWCNT): Multi-layered structure of hydrogen molecules

Wang, H., see Liu, H.

Wang, H., see Li, P.

Wang, H.-Y., see Tang, C.-M.

Wang, J., Xu, J., Lei, Q. \& Wang, $\mathrm{L}$., Influences of asymmetrically distributed defect states at rear c-Si/a-Si:H interface on performances of silicon heterojunction solar cells

Wang, J., see Tang, C.-M.

B27 (2013) 1350071

B27 (2013) 1330004

B27 (2013) 1350165

B27 (2013) 1350052

B27 (2013) 1350003

B27 (2013) 1350105 B27 (2013) 1350144

Wang, J.-H., see Lai, F.

Wang, J.-H., see Lai, F.

Wang, J.-S., see Liang, B.-L.

Wang, K. G., see Fa, K. S.

Wang, L. G. \& Huang, Y. L., The effect of gate on persistent current in mesoscopic open ring

Wang, L. J., see Zhu, J. L.

Wang, L., see Wang, J.

Wang, L., see Wang, X.

Wang, L.-N., see Zhou, H.-W.

Wang, P. X., see Chen, Z.

Wang, P., see Olorunsola, O.

Wang, P., see Zhen, H.-L.

Wang, R., see Chen, H.

Wang, S. D., see Mao, L.-F.

B27 (2013) $1350085 \quad$ Wang, S. Z., see Chen, T.-P.

Wang, S., see Yang, P.

Wang, T., see Wang, X.-Y.

Wang, W., see Chen, H.

Wang, W., see Chen, Z.

B27 (2013) 1350187 Wang, W., see Lei, M.

Wang, X. H., see Zhu, J. L.

Wang, X. \& Liang, S.-D., Topological effects of charge transfer in telomere G-quadruplex: Mechanism on telomerase activation and inhibition

B27 (2013) 1350132

B27 (2013) 1350040

B27 (2013) 1350126

B27 (2013) 1350127

B27 (2013) 1350134

B27 (2013) 1330006

B27 (2013) 1361005

B27 (2013) 1362023

B27 (2013) 1350132

B27 (2013) 1350033

B27 (2013) 1350080

B27 (2013) 1393001

B27 (2013) 1350175

B27 (2013) 1350029

B27 (2013) 1350149

B27 (2013) 1350172

B27 (2013) 1362009

B27 (2013) 1350150

B27 (2013) 1350196

B27 (2013) 1350149

B27 (2013) 1393001

B27 (2013) 1362019

B27 (2013) 1362023

B27 (2013) 1350001

B27 (2013) 1330016 
L., Finite-time chaos synchronization of a new hyperchaotic Lorenz system

Wang, X., see Nian, F.

Wang, X., see Nian, F.

Wang, X., see Nian, F.

Wang, X.-M., see Yuan, G.-Y.

Wang, X.-X., Qian, P., An, Z.-W., Li, Y.-P., Zhang, Z.-F., Li, J.C. \& Chen, N.-X., Theoretical study of phase forming, magnetization and lattice vibrations of $\mathrm{Fe}_{23-x} T_{x} B_{6}(T=C r, \mathrm{Mn}, \mathrm{Ni}, \mathrm{C})$, $\mathrm{Fe}_{23} \mathrm{C}_{6}, \mathrm{Fe}_{23} \mathrm{~B}_{6}$ and $\mathrm{Fe}_{23} \mathrm{CB}_{6}$

Wang, X.-Y. \& Tan, Y., A multipleplaintexts-oriented chaotic cryptosystem

Wang, X.-Y., Chen, F., Wang, T., $\mathrm{Xu}$, D. \& Ma, Y., Attack to an image encryption based on chaotic logistic map

Wang, X.-Y., Hu, Z.-W. \& Luo, C., Generalized synchronization of nonidentical fractionalorder chaotic systems

Wang, X.-Y., Jiang, S.-H. \& Luo, C., Adaptive synchronization of a novel hyperchaotic system with fully unknown parameters

Wang, X.-Y., Xu, B. \& Ma, Y., An improved chaotic masking scheme via system-alternating

Wang, X.-Y., Yang, Y.-H. \& Feng, M.-K., Synchronization between two different hyperchaotic systems with uncertain parameters

Wang, X.-Y., Zhao, G.-B. \& Yang, Y.-H., Diverse structure synchronization of fractional order hyper-chaotic systems

Wang, Y. G., see Shen, H. Q.

Wang, Y. \& Wei, B., Mixed-modal disk gas squeeze film theoretical and experimental analysis

Wang, Y., see Zhang, X.

Wang, Y., see Zhao, J.

Wang, Y.-S., see Cui, Y.-M.

Wang, Y.-Y., Yang, T., Tian, M. \& Liao, R.-J., The relationship between $D P$, fracture degree and mechanical strength of cellulose $I_{\beta}$ in insulation paper by molecular dynamic simulations

Wang, Z. O., see Mao, L.-F.
B27 (2013) 1350033
B27 (2013) 1350111
B27 (2013) 1350112
B27 (2013) 1350113
B27 (2013) 1350158

B27 (2013) 1350050

B27 (2013) 1350202

B27 (2013) 1350196

B27 (2013) 1350195

B27 (2013) 1350197

B27 (2013) 1350201

B27 (2013) 1350044

B27 (2013) 1350034 B27 (2013) 1350173

B27 (2013) 1350168 B27 (2013) 1350069 B27 (2013) 1350109 B27 (2013) 1361006

B27 (2013) 1350183 B27 (2013) 1350172
Wang, Z.-H., see Liu, Q.

Wang, Z.-H., see Liu, Q.

Weerasinghe, J., see Wang, D.

Wei, B., see Wang, Y.

Wei, J., see Ye, H.

Wei, X., see Ye, H.

Wei, Y., see Lu, X.

Wei, Z., see Lv, L.

Wei, Z., see Lv, L.

Werlang, T., Ribeiro, G. A. P. \& Rigolin, G., Interplay between quantum phase transitions and the behavior of quantum correlations at finite temperatures

Wu, C., Xie, S., Qi, F., Li, B., Wan, J. \& He, J., Effect of Corona discharges on the inception of positive upward leader-streamer system

Wu, H. \& Zhou, J., Optical properties of anthracene single crystals grown by a simple solution technique

Wu, J., see $\mathrm{Li}, \mathrm{M}$.

Wu, K., see Chen, T.-P.

Wu, M. S., see Liu, G.

Wu, T., Song, X.-K. \& Ye, L., The dynamics of geometric discord and its transfer in atom-cavityreservoir system

Wu, W., see Chung, T. F.

Wu, X., see Gui, Z.

Wu, Y. \& Chen, Y., Modified spinwave theory for the frustrated Heisenberg antiferromagnet on a square lattice

Wu, Y., see Zhao, J.

Wu, Y., see Zhao, J.

Xia, H.-P., see Lai, F.

Xia, H.-P., see Lai, F.

Xiao, T. T., Cai, C. Z., Tang, J. L. \& Huang, S. J., Modeling of transition temperature for pulsed laser deposition $\mathrm{NdBa}_{2} \mathrm{Cu}_{3} \mathrm{O}_{7-\delta}$ thin films via support vector regression

$\mathrm{Xiao}, \mathrm{X}$., see $\mathrm{Xu}, \mathrm{Z}$.

Xie, G.-Y. \& Liu, X.-R., Theoretical investigations of the EPR parameters of cubic $\mathrm{Yb}^{3+}$ center in PbS, PbSe and PbTe semiconductors

Xie, S., see Wu, C.

Xie, Y. J., see Chen, Z.

Xie, Y., Solving sine-Gordon type
B27 (2013) 1350013

B27 (2013) 1350014

B27 (2013) 1330016

B27 (2013) 1350168

B27 (2013) 1350144

B27 (2013) 1350144

B27 (2013) 1250212

B27 (2013) 1362036

B27 (2013) 1392002

B27 (2013) 1345032

B27 (2013) 1350165

B27 (2013) 1350022

B27 (2013) 1350146

B27 (2013) 1362009

B27 (2013) 1350188

B27 (2013) 1350136

B27 (2013) 1341002

B27 (2013) 1350007

B27 (2013) 1350021

B27 (2013) 1350109

B27 (2013) 1350110

B27 (2013) 1350126

B27 (2013) 1350127

B27 (2013) 1362040 B27 (2013) 1361003

B27 (2013) 1362032 B27 (2013) 1350165 B27 (2013) 1393001 
Author Index

equations by a modified variable separated ODE method

Xing, Q. K., see Fu, X. L.

Xing, X., see Lu, B.-S.

Xu, B., see Liu, G.

$\mathrm{Xu}$, B., see Wang, X.-Y.

$\mathrm{Xu}, \mathrm{D}$., see Wang, X.-Y.

$\mathrm{Xu}$, J. J., see Chen, Z.

$\mathrm{Xu}$, J., see Wang, J.

Xu, J.-S. \& Li, C.-F., Quantum discord under system-environment coupling: the two-qubit case

Xu, S., Song, X. K. \& Ye, L., Negativity and geometric quantum discord as indicators of quantum phase transition in the XY model with DzyaloshinskiiMoriya interaction

$\mathrm{Xu}, \mathrm{W}$., see Chen, $\mathrm{H}$.

Xu, X. \& Qian, T., Evaporative droplets in one-component fluids driven by thermal gradients on solid substrates

Xu, X.-F., see Yuan, H.-C.

Xu, X.-G., Meng, X.-H., Zhang, C.-Y. \& Gao, Y.-T., Analytical investigation of the CaudreyDodd-Gibbon-Kotera-Sawada equation using symbolic computation

Xu, Y.-J., see Yuan, H.-C.

$\mathrm{Xu}$, Z., Xiao X. \& Chen, Y., Spin-dependent electron transport through a three-terminal mesoscopic spin-orbit coupled systems

Xu, Z., see Tang, L.

$\mathrm{Xu}, \mathrm{Z}$., see Ye, H.

Xue, F.-X., see Cheng, Y.

Xue, Y., see Zhao, R.

Yan, Y., see Mao, L.-F.

Yang, F., see Lei, M.

Yang, H. X., see Zhu, J. L.

Yang, H., see Yang, P.

Yang, J., see Yang, P.

Yang, J.-J., see Li, T.-H.

Yang, K., Quantum liquid crystal phases in fermionic superfluids with pairing between fermion species of unequal densities

Yang, L. Q., Yang, X. S., Lv, L. \& Zhao, Y., Electrical transport and low-field magnetoresistance in $\mathrm{La}_{0.8} \mathrm{Sr}_{0.2} \mathrm{MnO}_{3} / 2 n$ composites
Yang, L., see Lv, L.

Yang, L., see Lv, L.

Yang, M., Yang, Q., Sima, W.-X., Yuan, T., Chen, L.-J. \& Huang, Y.-L., Reconstruction and identification and control method of ferroresonance overvoltage based on voltage time series

Yang, P., Li, X., Zhao, Y., Yang, H., Wang, S. \& Yang, J., Investigation for molecular attraction impact between contacting surfaces in micro-gears

Yang, Q., Liao, H., Cao, J. X., Ma, Y. \& Zhou, Y. C., Spontaneous polarization and its strain effects for orthorhombic and monoclinic $\mathrm{Bi}_{4} \mathrm{Ti}_{3} \mathrm{O}_{12}: \mathrm{A}$ first principles study

B27 (2013) 1350149

Yang, Q., see Yang, M.

Yang, Q.-Y., see Liang, B.-L.

Yang, R.-X., see Shao, C.-X.

B27 (2013) 1361008

B27 (2013) 1350120

B27 (2013) 1250124 B27 (2013) 1350120

B27 (2013) 1361003 B27 (2013) 1350092 B27 (2013) 1350144 B27 (2013) 1350130 B27 (2013) 1350129 B27 (2013) 1350172 B27 (2013) 1362019 B27 (2013) 1362023 B27 (2013) 1350150 B27 (2013) 1350150 B27 (2013) 1350148

B27 (2013) 1362001
Yang, S., see Tan, L.

Yang, S.-J., see Liu, Y.-K

Yang, T., see Wang, Y.-Y.

Yang, W.-L., see Zang, C.-L.

Yang, W.-X., see Wang, C.-Y.

Yang, X. S., see Yang, L. Q.

Yang, X., see Lei, M.

Yang, X., see Lei, M.

Yang, X., see Lv, L.

Yang, X., see Lv, L.

Yang, Y., Lin, M.-M. \& Duan, W.S., The anisotropic characters in two-dimensional lattice

Yang, Y.-H., see Wang, X.-Y.

Yang, Y.-H., see Wang, X.-Y.

Yang, Z., see Tang, L.

Yao, K. L., see Min, Y.

Yao, K., see Gong, P.

Yao, X.-X., see Liu, A.-P.

Yao, X.-X., see Sun, X.-S.

Yau, S.-T., see Lin, H.

Yavari, M., B- to Z-DNA transition probed by the Feoli's formalism for a Kirchhoff Model

Yazici, E., see Ozmetin, A. E.

Ye, F., see Lu, B.-S.

Ye, H., Zhang, R., Wang, D., Cui, Y., Wei, J., Wang, C., Xu, Z., Qu, S. \& Wei, X., Firstprinciples calculation of leadfree perovskite $\mathrm{SnTiO}_{3}$

Ye, L., see Wu, T.
Yang, S.-P., see Yuan, G.-Y.
B27 (2013) 1362036

B27 (2013) 1392002

B27 (2013) 1350006

B27 (2013) 1350150

B27 (2013) 1350138 B27 (2013) 1350006 B27 (2013) 1350134 B27 (2013) 1350052 B27 (2013) 1362017 B27 (2013) 1350070 B27 (2013) 1350158 B27 (2013) 1350183 B27 (2013) 1350176 B27 (2013) 1350187 B27 (2013) 1362012 B27 (2013) 1362018 B27 (2013) 1362019 B27 (2013) 1362036 B27 (2013) 1392002

B27 (2013) 1361010 B27 (2013) 1350034 B27 (2013) 1350044 B27 (2013) 1350092 B27 (2013) 1350081 B27 (2013) 1350087 B27 (2013) 1362031 B27 (2013) 1362030 B27 (2013) 1350107

B27 (2013) 1350121 B27 (2013) 1362016 B27 (2013) 1330012

B27 (2013) 1350144 B27 (2013) 1350136 
Author Index

Ye, L., see Xu, S.

Ye, L., see Yu, Y.

Yeo, J., see Kim, S.

Yin, Z.-Q., Geraci, A. A. \& Li, T., Optomechanics of levitated dielectric particles

Yinnon, T. A. \& Elia, V., Dynamics in perturbed very dilute aqueous solutions: theory and experimental evidence

Yoon, B.-G., see Choi, J.

You, Y.-W., see Hao, Q.-H.

Younas, S., see Alay-e-Abbas, S. M.

Young, S. L., see Chen, H. Z.

Young, S.-L., Chen, H.-Z., Kao, M.-C., Kung, C.-Y., Lin, C.-C., Lin, T.-T., Horng, L., Shih, Y.-T., Ou, C.-J. \& Lin, C.-H., Magnetic properties of La-doped and Cu-doped $\mathrm{ZnO}$ nanowires fabricated by hydrothermal method

Yu, B., see Li, L.

Yu, J., see Zang, C.-L.

Yu, J., see Zhu, J. L.

Yu, Q., see Chung, T. F.

Yu, W.-C., see Gu, S.-J.

Yu, Y. \& Ye, L., Proposal for a general quantum cloning machine via distant qubits in a quantum network

Yu. Beliayev, E., see Belevtsev, B. I.

Yu. Kuzminykh, N., see Aliev, M. A.

Yuan, A., see Zhang, X.

Yuan, G.-Y., Wang, X.-M., Wang, G.-R. \& Yang, S.-P., Effect of external periodic pulses on spiral dynamics and control of spiral waves

Yuan, H.-C., Xu, Y.-J., Chen, L. \& $\mathrm{Xu}, \mathrm{X} . \mathrm{F}$., Wigner function evolution of excited even and odd coherent state in thermal environment via thermo entangled approach

Yuan, T., see Yang, M.

Yuan, X.-H., see Zhang, L.

Yurtseven, H. \& Aslantaş, A., Calculation of the Raman frequencies at low pressures and temperatures ( $\sigma$-phase) solid nitrogen
B27 (2013) 1350074

B27 (2013) 1350154

B27 (2013) 1350010

B27 (2013) 1330018

B27 (2013) 1350005

B27 (2013) 1350062

B27 (2013) 1350011

B27 (2013) 1350100 B27 (2013) 1362003

B27 (2013) 1362006

B27 (2013) 1350104

B27 (2013) 1350176

B27 (2013) 1362023

B27 (2013) 1341002

B27 (2013) 1350106

B27 (2013) 1350154

B27 (2013) 1350026

B27 (2013) 1350189

B27 (2013) 1350069

B27 (2013) 1350158

B27 (2013) 1350120

B27 (2013) 1350006

B27 (2013) 1350101

B27 (2013) 1350125
Yurtseven, H. \& Saritaş, S., Thermodynamic quantities at high pressures in the $i$ and $\theta$ phases of solid nitrogen deduced by Raman frequency shifts for the internal modes in literature

Yurtseven, H. \& Çetinbaş İşeri, E., Raman study of benzene near the melting point

Yushankhai, V. \& Siurakshina, L., Analysis of crystal-field multiplets of $\mathrm{V}^{3+}$ Ion in perovskite oxides for resonant inelastic $X$ ray scattering spectroscopy

Zang, C.-L., Yu, J., Yang, W.-L., Feng, M. \& Kou, S.-P., Manipulating Schrödinger Cat state of an Ising chain via quantum tunneling effect

Zdravković, S., Satarić, M. V. \& Daniel, M., Kink solitons in DNA

Zhang, C. L. \& Hu, C. G., Roomtemperature ferromagnetic properties of Co-doped $\mathrm{ZnO}$ Naorod arrays

Zhang, C. Y., see Guo, C. Q.

Zhang, C.-H., Huang, S., Li, R.-Z., Shen, J. \& Chen, N.-X., Effects on mechanical properties of refractory metal doped $\mathrm{Ti}_{3} \mathrm{Al}$ alloy

Zhang, C.-Y., see Xu, X.-G.

Zhang, F., see Zhang, $X$.

Zhang, H., see Guo, C. Q.

Zhang, H., see Wang, H.

Zhang, L. J., see Mao, L.-F.

Zhang, L. \& Yuan, X.-H., Effect of periodically modulated noise on stochastic resonance in laser system

Zhang, L., Quantum size effect on the binding energies of groundstate and excited-state excitons in a Wurtzite $\mathrm{ZnO}$ nanowire

Zhang, L.-L., see Zhou, H.-W.

Zhang, M. \& Shi, J.-J., Donor bound excitons confined in Wurtzite InGaN/GaN quantum dot nanowire heterostructures

Zhang, M., see Lv, L.

Zhang, M., see Lv, L.

Zhang, Q., see Tong, M.-W.

Zhang, R., see Ye, H.
B27 (2013) 1350035

B27 (2013) 1350063

B27 (2013) 1350185

B27 (2013) 1350176

B27 (2013) 1350184

B27 (2013) 1362010

B27 (2013) 1362015

B27 (2013) 1350147

B27 (2013) 1250124

B27 (2013) 1350069

B27 (2013) 1362015

B27 (2013) 1350061

B27 (2013) 1350172

B27 (2013) 135010

B27 (2013) 1350032

B27 (2013) 1350080

B27 (2013) 1350186 B27 (2013) 1362036 B27 (2013) 1392002 B27 (2013) 1362008 B27 (2013) 1350144 
Zhang, S.-C., see Lv, M.

Zhang, X., Wang, Y., Zhang, F. \& Yuan, A., Density functional calculations on spectrum and electronic properties of $W_{n} C O$ $(n=1 \sim 6)$ clusters

Zhang, X., see Chen, Y.-M.

Zhang, X., see Duan, H.

Zhang, X., see Li, S.

Zhang, X.-H. \& Kou, S.-P., Mott insulator-superfluid transition in a generalized Bose-Hubbard model with topologically nontrivial flat-band

Zhang, Y. Z., see Wang, H. F.

Zhang, Y., see Jiang, J.

Zhang, Y.-P., see Lai, F.

Zhang, Y.-P., see Lai, F.

Zhang, Z. H., see Tao, H. L.

Zhang, Z., see Zhou, X

Zhang, Z.-F., see Wang, X.-X.

Zhao, G. L., see Malozovsky, Y.

Zhao, G.-B., see Wang, X.-Y.

Zhao, G.-L., see Tan, L.

Zhao, H., Chen, X., Lu, J. \& $\mathrm{Lu}, \mathrm{W}$. , Electronic properties of $\mathrm{HgTe}$ within different structures

Zhao, J. \& Wu, Y., Function projective synchronization of the chaotic systems with parameters unknown

Zhao, J., Wu, Y. \& Wang, Y., Adaptive function $Q-S$ synchronization of different chaotic (hyper-chaotic) systems

Zhao, L. F., see Jiang, J.

Zhao, Q., see Li, P.

Zhao, R., Pan, W. \& Xue, Y., Exploring the control of spiral waves and spatiotemporal chaos by stochastic and crosscoupling method

Zhao, Y., see Jiang, J.
B27 (2013) 1350177

B27 (2013) 1350069

B27 (2013) 1350095

B27 (2013) 1350178

B27 (2013) 1350161

B27 (2013) 1250214

B27 (2013) 1362029

B27 (2013) 1362022

B27 (2013) 1350126

B27 (2013) 1350127

B27 (2013) 1350078

B27 (2013) 1350118

B27 (2013) 1350050

B27 (2013) 1362013

B27 (2013) 1350034

B27 (2013) 1362017

B27 (2013) 1350086

B27 (2013) 1350110

B27 (2013) 1350109

B27 (2013) 1362022

B27 (2013) 1350039

B27 (2013) 1350129

B27 (2013) 1362022
Zhao, Y., see Lei, M.

Zhao, Y., see Lei, M.

Zhao, Y., see Lv, L.

Zhao, Y., see Lv, L.

Zhao, Y., see Yang, L. Q.

Zhao, Y., see Yang, P.

Zhen, H.-L., Tian, B., Wang, P., Liu, R.-X. \& Zhong, H., Soliton interaction of the ZakharovKuznetsov equations in plasma dynamics

Zheng, D. N., see Wang, H. F.

Zheng, P., see Nian, F.

Zhong, C. G., see Min, Y.

Zhong, H., see Zhen, H.-L.

Zhong, Y. \& Luo, H.-G., Orthogonal Dirac semimetal on honeycomb lattice

Zhou, H.-W., Wang, L.-N., Zhang, L.-L. \& Huang, Y.-N., Application of Reed-vibration mechanical spectroscopy for liquids in studying liquid crystallization

Zhou, J., see Wu, H.

Zhou, S. \& Sun, J.-X., Mobility dependent efficiencies of organic bulk-heterojunction solar cells with recombination via tail

Zhou, X. \& Zhang, Z., The relaxation of twisted chiral nematic liquid crystals with side-chain polymeric layer

Zhou, Y. C., see Yang, Q.

Zhu, C. Y., see Mao, L.-F

Zhu, J. L., Yang, H. X., Feng, S. M., Wang, L. J., Liu, Q. Q., Jin, C. Q., Wang, X. H., Li, L. T. \& Yu, J., The multiferroic properties of $\mathrm{Bi}\left(\mathrm{Fe}_{1 / 2} \mathrm{Cr}_{1 / 2}\right) \mathrm{O}_{3}$ compound

Zloshchastiev, K. G., see Sergi, A. Zou, J., see Liu, B.-Q.

Zverev, M. V., see Clark, J. W.
B27 (2013) 1362018

B27 (2013) 1362019

B27 (2013) 1362036

B27 (2013) 1392002

B27 (2013) 1362012

B27 (2013) 1350150

B27 (2013) 1350029

B27 (2013) 1362029

B27 (2013) 1350111

B27 (2013) 1350081

B27 (2013) 1350029

B27 (2013) 1361002

B27 (2013) 1350080

B27 (2013) 1350022

B27 (2013) 1350167

B27 (2013) 1350118

B27 (2013) 1350138

B27 (2013) 1350172

B27 (2013) 1362023 B27 (2013) 1350163 B27 (2013) 1345055 B27 (2013) 1347005 